\title{
SOME EXTREMAL FUNCTIONS IN FOURIER ANALYSIS
}

\author{
BY JEFFREY D. VAALER ${ }^{1}$
}

1. Introduction. In the late 1930 s A. Beurling observed that the entire function

$$
B(z)=\left(\frac{\sin \pi z}{\pi}\right)^{2}\left\{\sum_{n=0}^{\infty}(z-n)^{-2}-\sum_{m=-\infty}^{-1}(z-m)^{-2}+2 z^{-1}\right\}
$$

satisfies a simple and useful extremal property. We have

$$
\operatorname{sgn}(x) \leqslant B(x)
$$

for all real $x$ and

$$
\int_{-\infty}^{\infty} B(x)-\operatorname{sgn}(x) d x=1
$$

The function $B(z)$ is entire of exponential type $2 \pi$, and Beurling showed that if $F(z)$ is any entire function of exponential type $2 \pi$ satisfying $\operatorname{sgn}(x) \leqslant F(x)$ for all real $x$, then

$$
\int_{-\infty}^{\infty} F(x)-\operatorname{sgn}(x) d x \geqslant 1 .
$$

Moreover, he showed that there is equality in (1.4) if and only if $F(z)=B(z)$. As an application Beurling found an interesting inequality for almost periodic functions (we include it here in Theorem 15), but his results were never published.

In 1974 A. Selberg used the function $B(z)$ to obtain a sharp form of the large sieve inequality. Selberg noted that if $\chi_{E}(x)$ is the characteristic function of the interval $E=[\alpha, \beta]$ and

$$
C_{E}(z)=\frac{1}{2}\{B(\beta-z)+B(z-\alpha)\},
$$

then

$$
\chi_{E}(x) \leqslant C_{E}(x)
$$

Received by the editors November 14, 1983.

1980 Mathematics Subject Classification. Primary 42A10, 42A38; Secondary 10H30, 41A17.

${ }^{1}$ Research supported in part by National Science Foundation grant MCS-8303309. 
for all real $x$. For $x \neq \alpha$ and $x \neq \beta$ we have

$$
\chi_{E}(x)=\frac{1}{2}\{\operatorname{sgn}(\beta-x)+\operatorname{sgn}(x-\alpha)\},
$$

so (1.6) follows immediately from (1.2). Since $C_{E}(x)$ is continuous, the restrictions on $x$ can be removed. By using (1.3) and (1.5) Selberg observed that $C_{E}(x)$ is integrable along the real axis and

$$
\int_{-\infty}^{\infty} C_{E}(x)-\chi_{E}(x) d x=1
$$

Of course, $C_{E}(z)$ is entire of exponential type $2 \pi$, but now, for applications, it is usually more convenient to work with an equivalent property of the Fourier transform of $C_{E}$. Specifically, the Fourier transform

$$
\hat{C}_{E}(t)=\int_{-\infty}^{\infty} C_{E}(x) e(-t x) d x
$$

(where we write $e(u)=e^{2 \pi \imath u}$ ) is a continous function supported on $[-1,1]$.

To illustrate one of the simplest applications of Selberg's function, let $\lambda_{1}, \lambda_{2}, \ldots, \lambda_{N}$ be real numbers and

$$
f(x)=\sum_{n=1}^{N} a(n) e\left(\lambda_{n} x\right)
$$

an almost periodic trigonometric polynomial. Suppose that $\lambda_{1}, \lambda_{2}, \ldots, \lambda_{N}$ are well spaced in the sense that $\left|\lambda_{n}-\lambda_{m}\right| \geqslant 1$ whenever $n \neq m$. Using inequality (1.6) we have

$$
\begin{aligned}
\int_{\alpha}^{\beta}|f(x)|^{2} d x & \leqslant \int_{-\infty}^{\infty} C_{E}(x)|f(x)|^{2} d x \\
& =\sum_{n=1}^{N} \sum_{m=1}^{N} a(n) \overline{a(m)} \int_{-\infty}^{\infty} C_{E}(x) e\left(\left(\lambda_{n}-\lambda_{m}\right) x\right) d x \\
& =\sum_{n=1}^{N} \sum_{m=1}^{N} a(n) \overline{a(m)} \hat{C}_{E}\left(\lambda_{m}-\lambda_{n}\right)
\end{aligned}
$$

Now $\hat{C}_{E}\left(\lambda_{m}-\lambda_{n}\right)=0$ if $\left|\lambda_{m}-\lambda_{n}\right| \geqslant 1$, so all of the nondiagonal terms on the right of (1.8) are zero. It follows that

$$
\int_{\alpha}^{\beta}|f(x)|^{2} d x \leqslant \hat{C}_{E}(0) \sum_{n=1}^{N}|a(n)|^{2}=(\beta-\alpha+1) \sum_{n=1}^{N}|a(n)|^{2} .
$$

More generally, if $\left|\lambda_{n}-\lambda_{m}\right| \geqslant \delta>0$ for $n \neq m$, then an obvious change of variables in the previous argument leads to the upper bound

$$
\int_{\alpha}^{\beta}|f(x)|^{2} d x \leqslant\left(\beta-\alpha+\delta^{-1}\right) \sum_{n=1}^{N}|a(n)|^{2} .
$$

By modifying his original construction Selberg found an entire function $c_{E}(z)$ of exponential type $2 \pi$ which satisfies the minorizing inequality $c_{E}(x) \leqslant \chi_{E}(x)$ for all real $x$ and also

$$
\int_{-\infty}^{\infty} \chi_{E}(x)-c_{E}(x) d x=1
$$


Of course, this provides a lower bound which, when combined with (1.9), can be written as

$$
\int_{\alpha}^{\beta}|f(x)|^{2} d x=\left(\beta-\alpha+\theta \delta^{-1}\right) \sum_{n=1}^{N}|a(n)|^{2}
$$

with $-1 \leqslant \theta \leqslant 1$.

The identity (1.10) was also obtained by Montgomery and Vaughan [M-V] from a generalization of Hilbert's inequality. In fact, their form of Hilbert's inequality can also be established directly from Beurling's inequality (1.2) and knowledge of the Fourier transform of $B(x)-\operatorname{sgn}(x)$. We provide the details in Theorem 16.

The functions $c_{E}(z)$ and $C_{E}(z)$ occur as special cases of a general method for constructing entire functions of prescribed exponential type which minorize or majorize a given function of bounded variation. We describe this result in $\$ 4$.

Let

$$
S(x)=\sum_{n=M+1}^{M+N} a(n) e(n x)
$$

be a trigonometric polynomial with period 1 , and let $\xi_{1}, \xi_{2}, \ldots, \xi_{R}$ be real numbers which are well spaced modulo 1. Specifically, we suppose that $\left\|\xi_{r}-\xi_{s}\right\| \geqslant \delta>0$ for $r \neq s$, where $\|x\|$ is the distance from $x$ to the nearest integer. In its most basic setting the large sieve is an inequality of the form

$$
\sum_{r=1}^{R}\left|S\left(\xi_{r}\right)\right|^{2} \leqslant \Delta(N, \delta) \sum_{n=M+1}^{M+N}|a(n)|^{2} .
$$

By using the function $C_{E}(z)$ Selberg established (1.11) with $\Delta(N, \delta)=N-1$ $+\delta^{-1}$, which is sharp. An essentially equivalent bound with $\Delta(N, \delta)=N+\delta^{-1}$ was obtained at about the same time by Montgomery and Vaughan [Mon]. Selberg's proof of (1.11) is simple and direct. Let $F(z)$ be the entire function of exponential type $2 \pi \delta$ defined by $F(z)=C_{E}(\delta z)$, where $E=[\delta(M+1)$, $\delta(M+N)$ ]. It follows that $F(x) \geqslant 0$ for all real $x$ and $F(x) \geqslant 1$ for $M+1 \leqslant$ $x \leqslant M+N$. Since

$$
\hat{F}(t)=\delta^{-1} \hat{C}_{E}\left(\delta^{-1} t\right),
$$

we see that $\hat{F}$ is supported on $[-\delta, \delta]$ and $\hat{F}(0)=N-1+\delta^{-1}$. By a theorem of Fejer [Boa, pp. 124-126] there is an entire function $f(z)$ such that $F(x)=$ $|f(x)|^{2}$ for all real $x, f(x)$ is obviously in $L^{2}(\mathbf{R})$, and

$$
f(z)=\int_{-\delta / 2}^{\delta / 2} \hat{f}(t) e(z t) d t,
$$

that is, $\hat{f}$ is supported on $[-\delta / 2, \delta / 2]$. Now define

The identity

$$
S^{*}(x)=\sum_{n=M+1}^{M+N} a(n) f(n)^{-1} e(n x) .
$$

$$
S(x)=\int_{-\delta / 2}^{\delta / 2} \hat{f}(u) S^{*}(u+x) d u
$$


follows immediately. If we apply Cauchy's inequality to the right side of (1.12) we find that

$$
\begin{aligned}
\left|S\left(\xi_{r}\right)\right|^{2} & \leqslant \int_{-\delta / 2}^{\delta / 2}|\hat{f}(u)|^{2} d u \int_{-\delta / 2}^{\delta / 2}\left|S^{*}\left(u+\xi_{r}\right)\right|^{2} d u \\
& =\hat{F}(0) \int_{\xi_{r}-\delta / 2}^{\xi_{r}+\delta / 2}\left|S^{*}(u)\right|^{2} d u .
\end{aligned}
$$

Finally, we sum both sides of (1.13) over $r$ and use the well spacing of $\xi_{1}, \xi_{2}, \ldots, \xi_{R} \bmod 1$. In this way we obtain

$$
\begin{aligned}
\sum_{r=1}^{R}\left|S\left(\xi_{r}\right)\right|^{2} & \leqslant \hat{F}(0) \int_{0}^{1}\left|S^{*}(u)\right|^{2} d u \\
& =\left(N-1+\delta^{-1}\right) \sum_{n=M+1}^{M+N}|a(n)|^{2} F(n)^{-1} \\
& \leqslant\left(N-1+\delta^{-1}\right) \sum_{n=M+1}^{M+N}|a(n)|^{2}
\end{aligned}
$$

This method for proving the large sieve is implicit in [Sel, p. 215].

In view of the extremal property satisfied by Buerling's function $B(z)$, one might expect that a similar property would hold for the function $C_{E}(z)$. This is indeed the case, but only if the length $\beta-\alpha$ of the interval $E$ is an integer. Selberg has shown that if $F(z)$ is any entire function of exponential type $2 \pi$ which majorizes $\chi_{E}(x)$ along the real axis, then

$$
\int_{-\infty}^{\infty} F(x)-\chi_{E}(x) d x \geqslant 1
$$

provided that $\beta-\alpha$ is an integer. In this case $C_{E}(z)$ is clearly extremal; however, it is not unique. The set of all extremal functions for (1.15) was determined by Selberg (see $\left[\mathbf{G V}_{\mathbf{2}}\right.$, p. 289]). If $\beta-\alpha$ is not an integer, then inequality (1.15) is false in general. B. Logan [Log] has found the corresponding extremal function for $\beta-\alpha$ not an integer and established that it is unique.

Although Selberg's function $C_{E}(z)$ is not extremal for every interval $E$, it has proved to be a useful device for establishing several important inequalities. A further account of its applications in connection with the large sieve is contained in [Mon, $\mathbf{G V}_{\mathbf{1}}, \mathbf{G V}_{\mathbf{2}}$ and Sel]. Our purpose here is to give a more general discussion of the extremal problems which motivated the construction of $B(z)$ and $C_{E}(z)$ and to provide some additional applications.

Our notation for Fourier transforms, Fourier series, and convolutions follows that of Stein and Weiss [StW]. We say that a function $f: \mathbf{R} \rightarrow \mathbf{C}$ is normalized if

$$
\lim _{h \rightarrow 0+} \frac{1}{2}\{f(x+h)+f(x-h)\}=f(x)
$$

for every real $x$. An entire function $F(z), z=x+i y$, is said to have exponential type $\sigma \geqslant 0$ if, for every $\varepsilon>0$,

$$
|f(z)| \leqslant A(\varepsilon) e^{(\sigma+\varepsilon)|z|}
$$


for all $z$ and some positive constant $A(\varepsilon)$ which may depend on $\varepsilon$. We write $\|x\|$ for the distance from the real number $x$ to the nearest integer.

ACKNOWLedgement. The author wishes to thank Professors Arne Beurling and Atle Selberg for helpful conversations about this paper.

2. Special functions. We have noted that the function $B(x)$ majorizes $\operatorname{sgn}(x)$ and minimizes the integral on the left of (1.8). Before proving these facts about $B(x)$, we consider the following simpler question: How can $\operatorname{sgn}(x)$ be $a p$ proximated by an entire function $F(z)$ of exponential type $\sigma$ in such a way that the integral

$$
\int_{-\infty}^{\infty}|F(x)-\operatorname{sgn}(x)| d x
$$

is minimized? This problem can be reformulated in terms of Beurling's theory of minimal extrapolation [Beu], and a solution can be constructed from a general method of Sz. Nagy [SNa] (see also Shapiro [Sha, Chapter 7]). Here, however, we shall take a more direct approach which can be suitably modified to deal with $B(x)$ and the problem of majorizing $\operatorname{sgn}(x)$.

If $F(z)$ is an entire function of exponential type $\pi$ and $F(x)$ is bounded on $\mathbf{R}$, then $F(z)$ can be represented by the interpolation formula (Timan [Tim, p. 183] or Zygmund [Zyg, vol. II, p. 275])

$$
\begin{aligned}
F(z)= & \left(\frac{\sin \pi z}{\pi}\right) \\
& \cdot\left\{F(0) z^{-1}+\sum_{\substack{n=-\infty \\
n \neq 0}}^{\infty}(-1)^{n} F(n)\left((z-n)^{-1}+n^{-1}\right)+F^{\prime}(0)\right\} .
\end{aligned}
$$

This suggests that the special function

$$
G(z)=\left(\frac{\sin \pi z}{\pi}\right)\left\{\sum_{\substack{n=-\infty \\ n \neq 0}}^{\infty}(-1)^{n} \operatorname{sgn}(n)\left((z-n)^{-1}+n^{-1}\right)+\log 4\right\}
$$

which interpolates $\operatorname{sgn}(x)$ at the integers, should be a good approximation to $\operatorname{sgn}(x)$ on $\mathbf{R}$. In fact, $G(z)$ is the unique entire function which minimizes the integral (2.1) with $\sigma=\pi$. It will be clear from the following lemmas that our choice of $G^{\prime}(0)=\log 4$ is the right one.

LEMMA 1. The function $G(x)$ satisfies

$$
0 \leqslant \operatorname{sgn}(\sin \pi x)\{\operatorname{sgn}(x)-G(x)\} \leqslant\left|\frac{\sin \pi x}{\pi x}\right|(1+|x|)^{-1}
$$

for all real $x$.

Proof. We define functions $U_{N}(z)$ and $G_{N}(z)$ by

$$
U_{N}(z)=\left(\frac{\sin \pi z}{\pi}\right)\left\{z^{-1}+\sum_{\substack{n=-N \\ n \neq 0}}^{N}(-1)^{n}\left((z-n)^{-1}+n^{-1}\right)\right\}
$$


and

$$
\begin{array}{r}
G_{N}(z)=\left(\frac{\sin \pi z}{\pi}\right)\left\{\sum_{\substack{n=-N \\
n \neq 0}}^{N}(-1)^{n} \operatorname{sgn}(n)\left((z-n)^{-1}+n^{-1}\right)\right. \\
\left.-\sum_{\substack{m=-N \\
m \neq 0}}^{N}(-1)^{m} \operatorname{sgn}(m) m^{-1}\right\} .
\end{array}
$$

It follows that $U_{N}(z) \rightarrow 1$ and $G_{N}(z) \rightarrow G(z)$ uniformly on compact subsets of $\mathbf{C}$ as $N \rightarrow \infty$. From (2.5) and (2.6) we have

$$
\begin{aligned}
& \operatorname{sgn}(\sin \pi x)\left\{U_{2 N}(x)-G_{2 N}(x)\right\} \\
& \quad=\left|\frac{\sin \pi x}{\pi}\right|\left\{x^{-1}+2 \sum_{n=1}^{2 N}(-1)^{n}(x+n)^{-1}\right\} .
\end{aligned}
$$

Next we assume that $x>0$ and reorganize the sum on the right of (2.7). We find that

$$
\begin{aligned}
x^{-1}+2 & \sum_{n=1}^{2 N}(-1)^{n}(x+n)^{-1}=\sum_{n=0}^{2 N}(-1)^{n}(x+n)^{-1}+\sum_{m=1}^{2 N}(-1)^{m}(x+m)^{-1} \\
= & \sum_{l=0}^{N-1}\left\{(x+2 l)^{-1}(x+2 l+1)^{-1}-(x+2 l+1)^{-1}(x+2 l+2)^{-1}\right\} \\
& +(x+2 N)^{-1} \\
= & \sum_{n=0}^{2 N-1}(-1)^{n}(x+n)^{-1}(x+n+1)^{-1}+(x+2 N)^{-1} .
\end{aligned}
$$

Letting $N \rightarrow \infty$ we have

$$
\begin{aligned}
& \operatorname{sgn}(\sin \pi x)\{1-G(x)\} \\
& \quad=\left|\frac{\sin \pi x}{\pi}\right|\left\{\sum_{n=0}^{\infty}(-1)^{n}(x+n)^{-1}(x+n+1)^{-1}\right\} .
\end{aligned}
$$

Finally, we use the estimate

$$
0 \leqslant \sum_{n=0}^{\infty}(-1)^{n}(x+n)^{-1}(x+n+1)^{-1} \leqslant x^{-1}(x+1)^{-1}
$$

for the alternating series in (2.8) to deduce that (2.4) holds for $x>0$. Since the expressions in (2.4) are even functions, the inequality must also hold for $x<0$. The case $x=0$ is trivial, so the lemma is proved.

LEMMA 2. The function $I(z)=\frac{1}{2} G^{\prime}(z)$ satisfies

$$
I(x) \ll\left(1+x^{2}\right)^{-1}
$$

for all real $x$. Thus $I(x)$ is integrable, and its Fourier transform is given by

$$
\hat{I}(t)= \begin{cases}\pi t \cot \pi t & \text { if }|t| \leqslant 1 / 2 \\ 0 & \text { if }|t| \geqslant 1 / 2\end{cases}
$$


Proof. We write the function $G_{N}(z)$, defined by (2.6), in the form

$$
\begin{aligned}
G_{N}(z) & =\sum_{n=-N}^{N} \operatorname{sgn}(n) \frac{\sin \pi(z-n)}{\pi(z-n)} \\
& =\sum_{n=-N}^{N} \operatorname{sgn}(n) \int_{-1 / 2}^{1 / 2} e((z-n) t) d t .
\end{aligned}
$$

By applying $\frac{1}{2}(d / d z)$ to both sides of (2.11) and using the identity

$$
\sum_{n=-N}^{N} \operatorname{sgn}(n) e(-n t)=-i \cot \pi t+i\left\{\frac{\cos \pi(2 N+1) t}{\sin \pi t}\right\}
$$

we find that

$$
\begin{aligned}
\frac{1}{2} G_{N}^{\prime}(z)= & \int_{-1 / 2}^{1 / 2}\{\pi t \cot \pi t\} e(t z) d t \\
& -\int_{-1 / 2}^{1 / 2}\left\{\frac{\pi t}{\sin \pi t}\right\}(\cos \pi(2 N+1) t) e(t z) d t .
\end{aligned}
$$

As $N \rightarrow \infty$, the second integral on the right of (2.13) converges to zero by the Riemann-Lebesgue lemma. This establishes the representation

$$
I(z)=\int_{-1 / 2}^{1 / 2}\{\pi t \cot \pi t\} e(t z) d t
$$

Next we define $\psi(t)=\pi t \cot \pi t$ for $-1<t<1$ and integrate by parts twice in (2.14). We obtain

$$
I(z)=-(2 \pi z)^{-2}\left\{\pi^{2} \cot \pi z+\int_{-1 / 2}^{1 / 2} \psi^{\prime \prime}(t) e(t z) d t\right\},
$$

which proves the estimate (2.9). Of course, this also shows that $I(x)$ is integrable, and (2.10) then follows from (2.14) by the Fourier inversion formula.

COROLlary 3. The Fourier transform of the function $D(x)=G(x)-\operatorname{sgn}(x)$ is given by

$$
\hat{D}(t)= \begin{cases}0 & \text { if } t=0 \\ (\pi i t)^{-1}\{\hat{I}(t)-1\} & \text { if } t \neq 0 .\end{cases}
$$

Proof. Since $D$ is odd we may assume that $t \neq 0$. Then (2.15) is obtained from the formula

$$
\hat{I}(t)-1=\frac{1}{2} \int_{-\infty}^{\infty} e(-t x) d D(x)
$$

after an integration by parts.

THEOREM 4. If $F(z)$ is an entire function of exponential type $\sigma$, then

$$
\int_{-\infty}^{\infty}|F(x)-\operatorname{sgn}(x)| d x \geqslant \frac{\pi}{\sigma}
$$


for $\sigma>0$, and

$$
\int_{-\infty}^{\infty}|F(x)-\operatorname{sgn}(x)| d x=+\infty
$$

for $\sigma=0$. Moreover, there is equality in (2.16) if and only if $F(z)=G\left(\sigma \pi^{-1} z\right)$.

Proof. We begin by assuming that $\sigma>0$; then, by an obvious change of variables, we may assume that $\sigma=\pi$. Let $F(z)$ be an entire function of exponential type $\pi$ such that

$$
\int_{-\infty}^{\infty}|F(x)-\operatorname{sgn}(x)| d x<\infty .
$$

By Lemma 1 the function $\operatorname{sgn}(x)-G(x)$ is integrable, so, by the triangle inequality, $F(x)-G(x)$ is integrable. Since $F(z)-G(z)$ has exponential type $\pi$, it follows from a classical result of Polya and Plancheral [P-P] that $F^{\prime}(x)-G^{\prime}(x)$ is integrable. Finally, $G^{\prime}(x)$ is integrable by Lemma 2 , and thus $F^{\prime}(x)$ must be integrable. This also shows that $F(x)$ and $F(x)-\operatorname{sgn}(x)$ have bounded variation on $\mathbf{R}$.

For the remainder of the proof we write $\psi(x)=F(x)-\operatorname{sgn}(x)$ and $\varphi(x)$ $=\frac{1}{2} F^{\prime}(x)$. The Fourier transforms of $\psi$ and $\varphi$ are related by the identity

$$
\hat{\psi}(t)=(\pi i t)^{-1}\{\hat{\varphi}(t)-1\}
$$

for $t \neq 0$. This follows immediately from

$$
\hat{\varphi}(t)-1=\frac{1}{2} \int_{-\infty}^{\infty} e(-t x) d \psi(x)
$$

and an integration by parts. Since $\varphi(z)=\frac{1}{2} F^{\prime}(z)$ is an entire function of exponential type $\pi$, the transform $\hat{\varphi}(t)$ is continuous and supported on $\left[-\frac{1}{2}, \frac{1}{2}\right]$. Thus,

$$
\hat{\psi}(t)=-(\pi i t)^{-1}
$$

if $|t| \geqslant \frac{1}{2}$. Next we observe that $\operatorname{sgn}(\sin \pi x)$ has period 2 and the Fourier series expansion

$$
\operatorname{sgn}(\sin \pi x)=\frac{2}{\pi i} \sum_{k=-\infty}^{\infty}(2 k+1)^{-1} e\left(\left(k+\frac{1}{2}\right) x\right) .
$$

As $\operatorname{sgn}(\sin \pi x)$ is a normalized function of bounded variation on [0,2], this Fourier expansion converges at every point $x$ and the partial sums are uniformly bounded. Using (2.19) and (2.20) we obtain the lower bound

$$
\begin{aligned}
\int_{-\infty}^{\infty}|F(x)-\operatorname{sgn}(x)| d x & \geqslant\left|\int_{-\infty}^{\infty} \psi(x) \operatorname{sgn}(\sin \pi x) d x\right| \\
& =\left|\frac{2}{\pi i} \sum_{k=-\infty}^{\infty}(2 k+1)^{-1} \int_{-\infty}^{\infty} \psi(x) e\left(\left(k+\frac{1}{2}\right) x\right) d x\right| \\
& =\left|\frac{2}{\pi i} \sum_{k=-\infty}^{\infty}(2 k+1)^{-1} \hat{\psi}\left(-\left(k+\frac{1}{2}\right)\right)\right| \\
& =\frac{4}{\pi^{2}} \sum_{k=-\infty}^{\infty}(2 k+1)^{-2}=1 .
\end{aligned}
$$


It is clear from the lower bound in Lemma 1 that there is equality in (2.21) if $F(z)=G(z)$. On the other hand, if we assume that there is equality in (2.21) then $\{F(x)-\operatorname{sgn}(x)\} \operatorname{sgn}(\sin \pi x)$ does not change sign. Since $F(x)$ is continuous, we easily deduce that $F(n)=\operatorname{sgn}(n)$ at each integer $n$. From the interpolation formula (2.2) and (2.3) it follows that

$$
F(z)=G(z)+\beta \sin \pi z
$$

for some constant $\beta$. But we have already seen that $F(x)-G(x)$ is integrable; thus $\beta=0$.

If $F(z)$ has exponential type zero, then it is of exponential type $\sigma$ for every $\sigma>0$. Therefore (2.16) holds for every $\sigma>0$; hence $(2.17)$ is false.

We now turn our attention to the problem of majorizing $\operatorname{sgn}(x)$ by entire functions of exponential type. If $F(z)$ is entire of exponential type $2 \pi$, bounded on $\mathbf{R}$, and an odd function, then $F$ can be represented by the interpolation formula

$$
\begin{aligned}
F(z)= & \left(\frac{\sin \pi z}{\pi}\right)^{2} \\
& \cdot\left\{\sum_{m=-\infty}^{\infty} F(m)(z-m)^{-2}+\lim _{N \rightarrow \infty} \sum_{n=-N}^{N} F^{\prime}(n)(z-n)^{-1}\right\}
\end{aligned}
$$

This is a special case of a more general identity which we prove in $\S 3$. Of course, the advantage of (2.22) over (2.2) is that (2.22) interpolates both $F$ and $F^{\prime}$ at the integers. The price we pay for this is an increase in the exponential type from $\pi$ to $2 \pi$. In view of (2.22) we define three special functions, each having exponential type $2 \pi$, as follows:

$$
\begin{gathered}
H(z)=\left(\frac{\sin \pi z}{\pi}\right)^{2}\left\{\sum_{m=-\infty}^{\infty} \operatorname{sgn}(m)(z-m)^{-2}+2 z^{-1}\right\} \\
J(z)=\frac{1}{2} H^{\prime}(z), \quad \text { and } K(z)=\left(\frac{\sin \pi z}{\pi z}\right)^{2} .
\end{gathered}
$$

We note that $H(z)+K(z)$ is the function $B(z)$ defined by (1.1).

LEMMA 5. For all real $x$ we have

$$
|H(x)| \leqslant 1
$$

and

$$
|\operatorname{sgn}(x)-H(x)| \leqslant K(x) .
$$

Proof. It suffices to show that

$$
1-K(x) \leqslant H(x) \leqslant 1
$$

holds for $x>0$. The result will then follow easily from the observation that $\operatorname{sgn}(x)$ and $H(x)$ are odd functions.

From the identity

$$
\sum_{m=-\infty}^{\infty}(z-m)^{-2}=\left(\frac{\pi}{\sin \pi z}\right)^{2}
$$


we have (with $x>0$ )

$$
H(x)=1+\left(\frac{\sin \pi x}{\pi}\right)^{2}\left\{2 x^{-1}-x^{-2}-2 \sum_{m=1}^{\infty}(x+m)^{-2}\right\}
$$

By the arithmetic-geometric mean inequality,

$$
\begin{aligned}
x^{-2}+2 \sum_{m=1}^{\infty}(x+m)^{-2} & =\sum_{m=0}^{\infty}\left\{(x+m)^{-2}+(x+m+1)^{-2}\right\} \\
& \geqslant 2 \sum_{m=0}^{\infty}(x+m)^{-1}(x+m+1)^{-1}=2 x^{-1} .
\end{aligned}
$$

It follows that the inequality on the right of (2.25) holds for $x>0$. On the other hand,

$$
\sum_{m=1}^{\infty}(x+m)^{-2} \leqslant \sum_{m=0}^{\infty}(x+m)^{-1}(x+m+1)^{-1}=x^{-1},
$$

which, when combined with (2.26), confirms the inequality on the left of (2.25).

The bound (2.24) obviously implies Beurling's inequality (1.2) and also shows that $H(x)-\operatorname{sgn}(x)$ is integrable. To obtain (1.3) we note that

$$
\begin{aligned}
\int_{-\infty}^{\infty} B(x)-\operatorname{sgn}(x) d x & =\int_{-\infty}^{\infty} K(x) d x+\int_{-\infty}^{\infty} H(x)-\operatorname{sgn}(x) d x \\
& =\int_{-\infty}^{\infty} K(x) d x=1,
\end{aligned}
$$

since $H(x)-\operatorname{sgn}(x)$ is odd.

Next we consider the function $J(z)$.

THEOREM 6. The function $J(x)$ is integrable and satisfies

$$
J(x) \ll(1+|x|)^{-3}
$$

for all real $x$. The Fourier transform of $J(x)$ is given by

$$
\hat{J}(t)=\left\{\begin{array}{l}
1 \quad \text { if } t=0 \\
\pi t(1-|t|) \cot \pi t+|t| \quad \text { if } 0<|t|<1 \\
0 \quad \text { if } 1 \leqslant|t|
\end{array}\right.
$$

The function $\hat{J}(t)$ is even, nonnegative, continuously differentiable, and strictly decreasing on $[0,1]$.

Proof. Let

$$
H_{N}(z)=\left(\frac{\sin \pi z}{\pi}\right)^{2}\left\{\sum_{m=-N}^{N} \operatorname{sgn}(m)(z-m)^{-2}+2 z^{-1}\right\}
$$

so that

$$
\lim _{N \rightarrow \infty} H_{N}(z)=H(z) \text { and } \lim _{N \rightarrow \infty} \frac{1}{2} H_{N}^{\prime}(z)=J(z)
$$


uniformly on compact subsets of $\mathbf{C}$. From the identities

$$
K(z)=\int_{-1}^{1}(1-|t|) e(t z) d t
$$

and

$$
z K(z)=\frac{1}{2 \pi i} \int_{-1}^{1} \operatorname{sgn}(t) e(t z) d t
$$

we have

$$
\begin{aligned}
H_{N}(z)= & \sum_{m=-N}^{N} \operatorname{sgn}(m) K(z-m)+2 z K(z) \\
= & \int_{-1}^{1}(1-|t|)\left\{\sum_{m=-N}^{N} \operatorname{sgn}(m) e(-m t)\right\} e(t z) d t \\
& +\frac{1}{\pi i} \int_{-1}^{1} \operatorname{sgn}(t) e(t z) d t .
\end{aligned}
$$

As in our proof of Lemma 2, we apply $\frac{1}{2}(d / d z)$ to both sides of (2.31), use the identity (2.12) and the Riemann-Lebesgue lemma, and conclude that

$$
J(z)=\int_{-1}^{1}\{\pi t(1-|t|) \cot \pi t+|t|\} e(t z) d t .
$$

Let $\varphi(t)=\pi t(1-t) \cot \pi t+t$ for $-1<t<2$, with $\varphi$ defined by continuity at 0 and 1 . We then write (2.32) in the form

$$
J(z)=2 \int_{0}^{1} \varphi(t) \cos (2 \pi t z) d t
$$

and integrate by parts three times. This provides the representation

$$
J(z)=\frac{1}{(2 \pi z)^{3}}\left\{2 \int_{0}^{1} \varphi^{\prime \prime \prime}(t) \sin (2 \pi t z) d t-\frac{4 \pi^{2}}{3} \sin 2 \pi z\right\}
$$

and also shows that (2.27) must hold. It is clear from (2.27) that $J(x)$ is integrable and (2.28) follows from (2.32). The remaining properties attributed to $\hat{J}(t)$ are all easily verified.

COROLlaRY 7. The Fourier transform of the function $E(x)=H(x)-\operatorname{sgn}(x)$ is given by

$$
\hat{E}(t)= \begin{cases}0 & \text { if } t=0 \\ (\pi i t)^{-1}\{\hat{J}(t)-1\} & \text { if } t \neq 0 .\end{cases}
$$

We are now in a position to prove Beurling's result that the function $B(z)$ is extremal in inequality (1.4). In $\$ 3$ we show that the representation (2.22) also holds when $F(z)$ has exponential type $2 \pi$ and is integrable on $\mathbf{R}$. We use this fact in our proof of Beurling's theorem.

THEOREM 8. Let $F(z)$ be an entire function of exponential type $\sigma$ such that $F(x) \geqslant \operatorname{sgn}(x)$ for all real $x$. If $\sigma>0$ then

$$
\int_{-\infty}^{\infty} F(x)-\operatorname{sgn}(x) d x \geqslant \frac{2 \pi}{\sigma}
$$


if $\sigma=0$ then

$$
\int_{-\infty}^{\infty} F(x)-\operatorname{sgn}(x) d x=+\infty .
$$

Moreover, there is equality in (2.35) if and only if $F(z)=B\left(\sigma(2 \pi)^{-1} z\right)$.

Proof. If $\sigma>0$ then without loss of generality we may suppose that $\sigma=2 \pi$. We proceed exactly as in the proof of Theorem 4 , but use $H$ and $J$ in place of $G$ and $I$. We let $\psi(x)=F(x)-\operatorname{sgn}(x), \varphi(x)=\frac{1}{2} F^{\prime}(x)$, and show that $\varphi(x)$ is integrable. Again we obtain the identity

$$
\hat{\psi}(t)=(\pi i t)^{-1}\{\hat{\varphi}(t)-1\}
$$

if $t \neq 0$. Since $\hat{\varphi}(t)$ is now supported on the interval $[-1,1]$, we have

$$
\hat{\psi}(t)=-(\pi i t)^{-1}
$$

for $|t| \geqslant 1$.

At this point we use the Poisson summation formula (Zygmund [Zyg, Vol. I, p. 68]). Specifically, $\psi(x)$ is a normalized function of bounded variation; therefore

$$
\sum_{l=-\infty}^{\infty} \psi(x+l)=\sum_{m=-\infty}^{\infty} \hat{\psi}(m) e(m x)
$$

at each point $x$ (the symmetric partial sums of both series converge to the same value). From (2.37) we obtain

$$
\begin{aligned}
\sum_{m=-\infty}^{\infty} \hat{\psi}(m) e(m x) & =\hat{\psi}(0)-\sum_{\substack{m=-\infty \\
m \neq 0}}^{\infty}(\pi i m)^{-1} e(m x) \\
& = \begin{cases}\hat{\psi}(0)+2(x-[x]-1 / 2) & \text { if } x \notin \mathbf{Z}, \\
\hat{\psi}(0) & \text { if } x \in \mathbf{Z} .\end{cases}
\end{aligned}
$$

Now we observe that $\psi(x) \geqslant 0$, and, hence, the function (2.38) is nonnegative. This obviously implies that $\hat{\psi}(0) \geqslant 1$, which is exactly inequality (2.35).

If there is equality in $(2.35)$ - that is, if $\hat{\psi}(0)=1$-then

$$
\lim _{x \rightarrow 0+} \sum_{l=-\infty}^{\infty} \psi(x+l)=0 .
$$

Hence, $F(l+)=\operatorname{sgn}(l+)$, and therefore $F(l)=B(l)$, at each integer $l$. Since $F(x)$ majorizes $\operatorname{sgn}(x)$, we also conclude that $F^{\prime}(l)=0=B^{\prime}(l)$ at each integer $l \neq 0$. When we expand the entire function $F(z)-B(z)$, which is integrable on $\mathbf{R}$, by using (2.22), we find that

$$
F(z)-B(z)=\left(F^{\prime}(0)-2\right) z K(z) .
$$

Since $x K(x)$ is not integrable, we must have $F^{\prime}(0)=2$; hence, $F(z)=B(z)$.

Finally, if $\sigma=0$ we deduce that (2.36) holds exactly as in our proof of Theorem 4.

3. Interpolation formulas. The representation (2.22) is useful for constructing majorants because it allows us to control both $F(n)$ and $F^{\prime}(n)$. We now give a general account of this interpolation formula. Throughout this section $F(z)$ 
will denote an entire function of exponential type $\sigma$ with $\sigma \leqslant 2 \pi$. For $0<p \leqslant$ $\infty$ let $E^{p}$ be the set of those functions $F(z)$ which satisfy

$$
\int_{-\infty}^{\infty}|F(x)|^{p} d x<\infty \text { for } 0<p<\infty
$$

and

$$
\sup _{-\infty<x<\infty}|F(x)|<\infty \text { for } p=\infty .
$$

If $F(z)$ is in $E^{p}, 0<p<\infty$, then, by a result of Plancherel and Polya [P-P],

$$
\sum_{m=-\infty}^{\infty}|F(m)|^{p} \ll_{p} \int_{-\infty}^{\infty}|F(x)|^{p} d x
$$

and

$$
\int_{-\infty}^{\infty}\left|F^{\prime}(x)\right|^{p} d x \ll_{p} \int_{-\infty}^{\infty}|F(x)|^{p} d x .
$$

For $p=\infty$ the analogue of (3.2) is given by the classical inequality of Bernstein [Brn]: if $F(z)$ is in $E^{\infty}$ then

$$
\sup _{-\infty<x<\infty}\left|F^{\prime}(x)\right| \leqslant 2 \pi\left\{\sup _{-\infty<x<\infty}|F(x)|\right\} \text {. }
$$

By the Paley-Weiner theorem, $F(z)$ is in $E^{2}$ if and only if

$$
F(z)=\int_{-1}^{1} \hat{F}(t) e(t z) d t
$$

with $\hat{F}(t)$ in $L^{2}([-1,1])$. Of course, $\hat{F}(t)$ is the Fourier transform of $F$, defined for almost all $t$ by

$$
\hat{F}(t)=\lim _{T \rightarrow \infty} \int_{-T}^{T} F(x) e(-t x) d x
$$

and $\hat{F}(t)=0$ for almost all $t$, with $|t| \geqslant 1$.

THEOREM 9. Let $F(z)$ be an entire function in $E^{p}$ for some finite $p$. Then

$$
F(z)=\left(\frac{\sin \pi z}{\pi}\right)^{2}\left\{\sum_{m=-\infty}^{\infty} F(m)(z-m)^{-2}+\sum_{n=-\infty}^{\infty} F^{\prime}(n)(z-n)^{-1}\right\},
$$

where the expression on the right of (3.5) converges uniformly on compact subsets of $\mathbf{C}$.

If $p=2$ the Fourier transform $\hat{F}(t)$ occurring in (3.4) has the form

$$
\hat{F}(t)=(1-|t|) u_{F}(t)+(2 \pi i)^{-1} \operatorname{sgn}(t) v_{F}(t)
$$

for almost all $t$ in $[-1,1]$, where $u_{F}$ and $v_{F}$ are periodic functions in $L^{2}([0,1])$ with period 1 and Fourier series expansions

$$
u_{F}(t)=\sum_{m=-\infty}^{\infty} F(m) e(-m t)
$$

and

$$
v_{F}(t)=\sum_{n=-\infty}^{\infty} F^{\prime}(n) e(-n t)
$$


If $p=1$ then (3.7) and (3.8) are absolutely convergent, $u_{F}$ and $v_{F}$ are continuous, and (3.6) holds for all $t$ in $[-1,1]$. In particular,

$$
\sum_{m=-\infty}^{\infty} F(m)=u_{F}(0)=\hat{F}(0)
$$

and

$$
\sum_{n=-\infty}^{\infty} F^{\prime}(n)=v_{F}(0)=0 .
$$

Proof. To begin with, we suppose that $p=2$, so $F(z)$ is given by (3.4). For $0 \leqslant t<1$ we define

$$
u_{F}(t)=\hat{F}(t)+\hat{F}(t-1)
$$

and

$$
v_{F}(t)=2 \pi i\{t \hat{F}(t)+(t-1) \hat{F}(t-1)\} .
$$

We then extend the domain of $u_{F}$ and $v_{F}$ to $\mathbf{R}$ by requiring that both functions have period 1 . Since $\hat{F}$ is in $L^{2}([-1,1])$, it is clear that $u_{F}$ and $v_{F}$ are in $L^{2}([0,1])$. The identity (3.6) follows easily from (3.11), (3.12), and the periodicity of $u_{F}$ and $v_{F}$. To obtain the expansions (3.7) and (3.8), we note that

$$
F(n)=\int_{0}^{1}\{\hat{F}(t)+\hat{F}(t-1)\} e(t n) d t=\int_{0}^{1} u_{F}(t) e(t n) d t
$$

and

$$
\begin{aligned}
F^{\prime}(n) & =\int_{-1}^{1} 2 \pi i t \hat{F}(t) e(t n) d t \\
& =\int_{0}^{1} 2 \pi i\{t \hat{F}(t)+(t-1) \hat{F}(t-1)\} e(t n) d t \\
& =\int_{0}^{1} v_{F}(t) e(t n) d t
\end{aligned}
$$

for each integer $n$. Thus, $F(n)$ and $F^{\prime}(n)$ are the Fourier coefficients of $u_{F}$ and $v_{F}$, respectively.

Next we apply the Fourier transform identities (2.29) and (2.30). It follows that for each positive integer $N$,

$$
\begin{aligned}
& \left(\frac{\sin \pi z}{\pi}\right)^{2}\left\{\sum_{m=-N}^{N} F(m)(z-m)^{-2}+\sum_{n=-N}^{N} F^{\prime}(n)(z-n)^{-1}\right\} \\
& =\int_{-1}^{1}\left\{(1-|t|) u_{F}(t, N)+(2 \pi i)^{-1} \operatorname{sgn}(t) v_{F}(t, N)\right\} e(t z) d t
\end{aligned}
$$

where

$$
u_{F}(t, N)=\sum_{m=-N}^{N} F(m) e(-m t)
$$

and

$$
v_{F}(t, N)=\sum_{n=-N}^{N} F^{\prime}(n) e(-n t)
$$


Since the sequences $F(m)$ and $F^{\prime}(n)$ are square summable, the left side of (3.13) converges uniformly on compact subsets of $\mathbf{C}$ as $N \rightarrow \infty$. On the right side of (3.13) we have $u_{F}(t, N) \rightarrow u_{F}(t)$ and $v_{F}(t, N) \rightarrow v_{F}(t)$ in $L^{2}$-norm. This is all we need to establish the representation (3.5).

If $p=1$ then (3.1) and (3.2) imply that $u_{F}(t)$ and $v_{F}(t)$ have absolutely convergent Fourier series. Thus, we may take $u_{F}$ and $v_{F}$ to be continuous periodic functions. Since $\hat{F}(t)$ is now continuous and supported on $[-1,1]$, the identity (3.6) must hold for all $t$ in $[-1,1]$. If we let $t=0$, then (3.9) and (3.10) follow immediately.

Finally, we must show that (3.5) holds if $F(z)$ is an entire function in $E^{p}$ with $2<p<\infty$. We accomplish this by considering the entire function

$$
R(z)= \begin{cases}z^{-1}(F(z)-F(0)) & \text { if } z \neq 0 \\ F^{\prime}(0) & \text { if } z=0\end{cases}
$$

and its derivative

$$
R^{\prime}(z)= \begin{cases}z^{-2}\left(F(0)+z F^{\prime}(z)-F(z)\right) & \text { if } z \neq 0 \\ \frac{1}{2} F^{\prime \prime}(0) & \text { if } z=0\end{cases}
$$

Since $R(z)$ is in $E^{2}$, by the first part of our proof we have

$$
\begin{aligned}
R(z)= & \lim _{N \rightarrow \infty}\left(\frac{\sin \pi z}{\pi}\right)^{2} \\
& \cdot\left\{\sum_{m=-N}^{N} R(m)(z-m)^{-2}+\sum_{n=-N}^{N} R^{\prime}(n)(z-n)^{-1}\right\}
\end{aligned}
$$

uniformly on compact subsets of $\mathbf{C}$. Next we multiply both sides of (3.16) by $z$ and use (3.14) and (3.15). After a brief computation we find that

(3.17) $\quad F(z)-F(0)$

$$
\begin{array}{r}
=\lim _{N \rightarrow \infty}\left(\frac{\sin \pi z}{\pi}\right)^{2}\left\{\sum_{m=-N}^{N} F(m)(z-m)^{-2}+\sum_{n=-N}^{N} F^{\prime}(n)(z-n)^{-1}\right. \\
\left.+\sum_{k=-N}^{N} R^{\prime}(k)-F(0) \sum_{l=-N}^{N}(z-l)^{-2}\right\} .
\end{array}
$$

As the identity

$$
\sum_{l=-\infty}^{\infty}(z-l)^{-2}=\left(\frac{\pi}{\sin \pi z}\right)^{2}
$$

is well known; all that remains is to show that

We have

$$
\lim _{N \rightarrow \infty} \sum_{k=-N}^{N} R^{\prime}(k)=0 \text {. }
$$

$$
\begin{aligned}
\int_{1 \leqslant|x|}\left|R^{\prime}(x)\right| d x \leqslant & \int_{1 \leqslant|x|}|x|^{-2}|F(0)-F(x)| d x \\
& +\int_{1 \leqslant|x|}|x|^{-1}\left|F^{\prime}(x)\right| d x .
\end{aligned}
$$


The first integral on the right of (3.18) is obviously finite. The second integral is also finite because $F^{\prime}(x)$ is in $L^{p}(\mathbf{R})$ by (3.2). Thus, $R^{\prime}(z)$ is in $E^{1}$. Since we have already established (3.10) for functions in $E^{1}$, we obtain

$$
\sum_{k=-\infty}^{\infty} R^{\prime}(k)=\int_{-\infty}^{\infty} R^{\prime}(x) d x=0
$$

This completes our proof.

THEOREM 10. Let $F(z)$ be an entire function of exponential type $\sigma$ with $\sigma \leqslant 2 \pi$, let $R(z)$ be defined by (3.14), and suppose that $R(z)$ is in $E^{p}$ for some finite $p$. Then

$$
\begin{gathered}
F(z)=\left(\frac{\sin \pi z}{\pi}\right)^{2}\left\{\sum_{m=-\infty}^{\infty} F(m)(z-m)^{-2}+F^{\prime}(0) z^{-1}\right. \\
\left.\quad+\sum_{\substack{n=-\infty \\
n \neq 0}}^{\infty} F^{\prime}(n)\left\{(z-n)^{-1}+n^{-1}\right\}+A_{F}\right\},
\end{gathered}
$$

where the expression on the right of (3.19) converges uniformly on compact subsets of $\mathbf{C}$, and $A_{F}$ is a constant given by

$$
A_{F}=\frac{1}{2} F^{\prime \prime}(0)+\sum_{\substack{n=-\infty \\ n \neq 0}}^{\infty}(F(0)-F(n)) n^{-2} \text {. }
$$

Proof. Since $R(z)$ is in $E^{p}$, we may apply Theorem 9. As in the proof of that result, we find that (3.16) and (3.17) holds. Now, however, we reorganize (3.17) and use (3.15) to obtain

$F(z)=\lim _{N \rightarrow \infty}\left(\frac{\sin \pi z}{\pi}\right)^{2}$

$$
\text { (3.21) } \begin{array}{r}
\left\{\sum_{m=-N}^{N} F(m)(z-m)^{-2}+F^{\prime}(0) z^{-1}+\sum_{\substack{n=-N \\
n \neq 0}}^{N} F^{\prime}(n)\left\{(z-n)^{-1}+n^{-1}\right\}\right. \\
\left.+\frac{1}{2} F^{\prime \prime}(0)+\sum_{\substack{k=-N \\
k \neq 0}}^{N}(F(0)-F(k)) k^{-2}\right\} .
\end{array}
$$

If $0<p<q<\infty$, then $E^{p} \subseteq E^{q}$. Thus, we may assume without loss of generality that $1<p<\infty$. It follows that

$$
\begin{aligned}
\sum_{\substack{m=-\infty \\
m \neq 0}}^{\infty}\left|F(m) m^{-1}\right|^{p} & =\sum_{\substack{m=-\infty \\
m \neq 0}}^{\infty}\left|R(m)+F(0) m^{-1}\right|^{p} \\
& \leqslant 2^{p} \sum_{\substack{m=-\infty \\
m \neq 0}}^{\infty}\left\{|R(m)|^{p}+\left|F(0) m^{-1}\right|^{p}\right\}<\infty
\end{aligned}
$$


and

$$
\begin{aligned}
\sum_{\substack{n=-\infty \\
n \neq 0}}^{\infty}\left|F^{\prime}(n) n^{-1}\right|^{p} & =\sum_{\substack{n=-\infty \\
n \neq 0}}^{\infty}\left|R^{\prime}(n)+R(n) n^{-1}\right|^{p} \\
& \leqslant 2^{p} \sum_{\substack{n=-\infty \\
n \neq 0}}^{\infty}\left\{\left|R^{\prime}(n)\right|^{p}+\left|R(n) n^{-1}\right|^{p}\right\}<\infty
\end{aligned}
$$

For the series defining $A_{F}$ we also have

$$
\sum_{\substack{k=-\infty \\ k \neq 0}}^{\infty}\left|(F(0)-F(k)) k^{-2}\right|=\sum_{\substack{k=-\infty \\ k \neq 0}}^{\infty}|R(k)||k|^{-1}<\infty .
$$

Estimates (3.22)-(3.24), together with (3.21), show that the right side of (3.19) converges uniformly on compact subsets, with $A_{F}$ given by the absolutely convergent series (3.20).

We note that if $F(z)$ is odd and bounded on $\mathbf{R}$, then $A_{F}=0$ and (3.19) reduces to (2.22).

4. Majorizing functions of bounded variation. Let $f: \mathbf{R} \rightarrow \mathbf{C}$ be a normalized function of bounded variation. The results of $\$ 2$ can be applied in a simple way to prove approximation and majorization theorems for such a function $f$. We use the following notation: If $\delta>0$ we write $F_{\delta}(x)=\delta F(\delta x)$. If $F(x)$ is integrable we have $\hat{F}_{\delta}(t)=\hat{F}\left(\delta^{-1} t\right)$. In this case we also define the convolutions

$$
f * F(x)=\int_{-\infty}^{\infty} f(\xi) F(x-\xi) d \xi
$$

and

$$
(d f) * F(x)=\int_{-\infty}^{\infty} F(x-\xi) d f(\xi)
$$

For entire functions $F(z)$ of exponential type $\sigma$ with $F(x)$ integrable, it is easy to verify that $f * F(z)$ and $(d f) * F(z)$ have exponential type at most $\sigma$. We denote the total variation of $f$ on $(-\infty, x]$ by $V_{f}(x)$ and let $V_{f}=\lim _{x \rightarrow+\infty} V_{f}(x)$.

THEOREM 11. The entire function $f * I_{\delta}(z)$ has exponential type at most $\pi \delta$ and satisfies

$$
\int_{-\infty}^{\infty}\left|f(x)-f * I_{\delta}(x)\right| d x \leqslant(2 \delta)^{-1} V_{f} .
$$

The entire functions $f * J_{\delta}(z)$ and $\left(d V_{f}\right) * K_{\delta}(z)$ have exponential type at most $2 \pi \delta$ and satisfy

$$
\left|f(x)-f * J_{\delta}(x)\right| \leqslant(2 \delta)^{-1}\left(d V_{f}\right) * K_{\delta}(x)
$$

for all real $x$. 
Proof. Let $x$ be a point at which $f$ is continuous. Then

$$
\begin{aligned}
f(x)= & \frac{1}{2} \int_{-\infty}^{\infty} f(x-\xi) d\{\operatorname{sgn}(\delta \xi)\}=\int_{-\infty}^{\infty} f(x-\xi) I_{\delta}(\xi) d \xi \\
& +\frac{1}{2} \int_{-\infty}^{\infty} f(x-\xi) d\{\operatorname{sgn}(\delta \xi)-G(\delta \xi)\} \\
= & f * I_{\delta}(x)+\frac{1}{2} \int_{-\infty}^{\infty}\{\operatorname{sgn}(\delta(x-\xi))-G(\delta(x-\xi))\} d f(\xi)
\end{aligned}
$$

after integrating by parts. Since $f$ is continuous at almost all $x$, we have

$$
\begin{aligned}
\int_{-\infty}^{\infty} & \left|f(x)-f * I_{\delta}(x)\right| d x \\
& \leqslant \frac{1}{2} \int_{-\infty}^{\infty} \int_{-\infty}^{\infty}|\operatorname{sgn}(\delta(x-\xi))-G(\delta(x-\xi))| d V_{f}(\xi) d x \\
& =(2 \delta)^{-1} V_{f},
\end{aligned}
$$

by using the case of equality in (2.16).

In like manner we obtain

$$
f(x)-f * J_{\delta}(x)=\frac{1}{2} \int_{-\infty}^{\infty}\{\operatorname{sgn}(\delta(x-\xi))-H(\delta(x-\xi))\} d f(\xi)
$$

at each point $x$ where $f$ is continuous. By combining (2.24) and (4.3) we find that

$$
\begin{aligned}
\left|f(x)-f * J_{\delta}(x)\right| & \leqslant \frac{1}{2 \delta} \int_{-\infty}^{\infty} K_{\delta}(x-\xi) d V_{f}(\xi) \\
& =\frac{1}{2 \delta}\left(d V_{f}\right) * K_{\delta}(x) .
\end{aligned}
$$

Since $f$ satisfies the normalization (1.16), it is trivial to extend (4.4) to points of discontinuity by considering left- and right-hand limits. This completes the proof.

The Fourier transforms of the integrable functions

$$
\Psi_{\delta}(f, x)=f(x)-f * I_{\delta}(x)
$$

and

$$
\Phi_{\delta}(f, x)=f(x)-f * J_{\delta}(x)
$$

can be computed exactly as in our proof of Corollary 3 . We find that

$$
\hat{\Psi}_{\delta}(f, t)=\widehat{d f}(t)\left(\frac{1-\hat{I}\left(\delta^{-1} t\right)}{2 \pi i t}\right)
$$

and

$$
\hat{\Phi}_{\delta}(f, t)=\widehat{d f}(t)\left(\frac{1-\hat{J}\left(\delta^{-1} t\right)}{2 \pi i t}\right)
$$

for $t \neq 0$, and $\hat{\Psi}_{\delta}(f, 0)=\hat{\Phi}_{\delta}(f, 0)=0$. Here we have written

$$
\widehat{d f}(t)=\int_{-\infty}^{\infty} e(-t x) d f(x)
$$

for the Fourier-Stieltjes transform of $f$. 
For real-valued functions $f,(4.2)$ provides the following majorization.

COROLLARY 12. If $f$ is real valued then the entire function

$$
M(f, \delta, z)=f * J_{\delta}(z)+(2 \delta)^{-1}\left(d V_{f}\right) * K_{\delta}(z)
$$

has exponential type at most $2 \pi \delta$, satisfies $f(x) \leqslant M(f, \delta, x)$ for all real $x$, and

$$
\int_{-\infty}^{\infty} M(f, \delta, x)-f(x) d x=(2 \delta)^{-1} V_{f} .
$$

Since the total variation of $\operatorname{sgn}(x)$ is 2 , Theorem 4 shows that there is equality in (4.1) when $f(x)=\operatorname{sgn}(x)$.

If $f(x)$ is real valued and integrable, then $M(f, \delta, x)$ is integrable. A straightforward computation shows that

$$
\hat{M}(f, \delta, t)=\hat{f}(t) \hat{J}_{\delta}(t)+(2 \delta)^{-1} \widehat{d V}_{f}(t) \hat{K}_{\delta}(t)
$$

for all real $t$. Thus $\hat{M}(f, \delta, t)$ is supported on $[-\delta, \delta]$. In particular, if $f(x)=\chi_{E}(x)$ for some interval $E=[\alpha, \beta]$, then the right side of (4.8) is

$$
\begin{aligned}
\frac{1}{2} \int_{\alpha}^{\beta} \delta H^{\prime}(\delta(x-\xi)) & d \xi+\frac{1}{2 \delta}\left\{K_{\delta}(x-\alpha)+K_{\delta}(x-\beta)\right\} \\
= & \frac{1}{2}\{H(\delta(x-\alpha))-H(\delta(x-\beta))\} \\
& +\frac{1}{2}\{K(\delta(x-\alpha))+K(\delta(x-\beta))\} \\
= & \frac{1}{2}\{B(\delta(\beta-x))+B(\delta(x-\alpha))\} .
\end{aligned}
$$

Thus Corollary 12 generalizes Selberg's construction (1.5).

While the function $M(f, \delta, z)$ majorizes $f(x)$ on $\mathbf{R}$, we cannot, in general, expect $M(f, \delta, z)$ to be an extremal function which minimizes the integral (4.9). For certain special functions $f$, such an extreme majorant can be obtained by simply constructing, by means of Theorems 9 or 10 , an entire function of exponential type which interpolates $f(x)$ and $f^{\prime}(x)$ at the integers. This approach has been carried out by Graham and Vaaler $\left[\mathbf{G V}_{2}\right]$. An alternative method is illustrated by the entire functions $\tau_{k}(z)=z^{2 k-1} H(z)$, where $k=0,1,2, \ldots$ It is clear that each $\tau_{k}(z)$ has exponential type $2 \pi$ and, by (2.23), satisfies $\tau_{k}(x) \leqslant|x|^{2 k-1}$ for all real $x$. For $k=0$ this can be improved to $\tau_{0}(x) \leqslant \min \left\{2,|x|^{-1}\right\}$. Among all entire functions of type $2 \pi$ which minorize $\min \left\{2,|x|^{-1}\right\}$, the function $\tau_{0}(z)$ can be shown to be extremal in the sense that $\int_{-\infty}^{\infty} \min \left\{2,|x|^{-1}\right\}-\tau_{0}(x) d x$ is minimized. We note that $\tau_{0}(z)$ interpolates the values of $\min \left\{2,|x|^{-1}\right\}$ and its derivative at the integers. For $k=1,2, \ldots$ the nonnegative functions $|x|^{2 k-1}-\tau_{k}(x)$ are no longer integrable.

5. The Berry-Esseen inequality. Let $f(x)$ and $g(x)$ be normalized probability distribution functions. An important problem in probability theory is to estimate the difference $f(x)-g(x)$ by an expression depending on $\widehat{d f}(t)$ $-\widehat{d g}(t)$ for values of $t$ restricted to an interval $|t| \leqslant \delta$. For example, if $g(x)$ has 
a density function bounded from above by the positive constant $M$, then

$$
\sup _{x}|f(x)-g(x)| \leqslant c_{1} \delta^{-1} M+c_{2} \int_{-\delta}^{\delta}|t|^{-1}|\widehat{d f}(t)-\widehat{d g}(t)| d t
$$

for certain positive constants $c_{1}$ and $c_{2}$ and all $\delta>0$. With our definition of the Fourier-Stieltjes transform, the usual proofs of (5.1) give $c_{1}=12 \pi^{-2}$ and $c_{2}=\pi^{-1}$ (Feller [Fel], Loève [Loe]). Earlier versions of this inequality were used by Berry [Brr] and Esseen [Ess] in order to estimate the rate of convergence in the central limit theorem. Since their work has appeared, there have been many refinements. We note in particular the papers of Zolotarev $\left[\mathbf{Z o}_{\mathbf{1}}-\mathbf{Z o}_{\mathbf{3}}\right]$, van Beek [vBe], and H. Prawitz $\left[\mathbf{P r}_{\mathbf{1}}-\mathbf{P r}_{\mathbf{5}}\right]$.

Inequalities sharper than (5.1) can be obtained directly from Corollary 12 . Here we give two results of this type which are essentially the same as those obtained by Prawitz $\left[\operatorname{Pr}_{1}\right]$. For the purpose of this application, Prawitz obtained inequality (2.24) in a manner quite different from ours.

THEOREM 13. Let $f(x)$ and $g(x)$ be probability distribution functions with Fourier-Stieltjes transforms defined by (4.7). Suppose $t^{-1}\{\widehat{d f}(t)-\widehat{d g}(t)\}$ is integrable on a neighborhood of zero and $g$ has a density function $g^{\prime}(x)$ bounded from above by $M$. Then

$$
\begin{aligned}
\mid f(x)-g(x)- & \int_{-\delta}^{\delta} \hat{J}\left(\delta^{-1} t\right)(2 \pi i t)^{-1}\{\widehat{d f}(t)-\widehat{d g}(t)\} e(x t) d t \mid \\
& \leqslant \frac{1}{2 \delta}\left\{M+\int_{-\delta}^{\delta} \hat{K}\left(\delta^{-1} t\right)\{\widehat{d f}(t)-\widehat{d g}(t)\} e(x t) d t\right\}
\end{aligned}
$$

for all real $x$ and all $\delta>0$.

Proof. Since $f(x)$ is increasing, (4.7) takes the form

$$
f(x) \leqslant f * J_{\delta}(x)+(2 \delta)^{-1}(d f) * K_{\delta}(x) .
$$

It follows that

$$
\begin{aligned}
f(x)-g(x) \leqslant & (f-g) * J_{\delta}(x)+(2 \delta)^{-1}(d f-d g) * K_{\delta}(x) \\
& +g * J_{\delta}(x)+(2 \delta)^{-1}(d g) * K_{\delta}(x)-g(x)
\end{aligned}
$$

In the first term on the right of (5.3) we have

$$
\begin{aligned}
(f-g) * J_{\delta}(x)= & \int_{-\infty}^{\infty}\{f(\xi)-g(\xi)\} J_{\delta}(x-\xi) d \xi \\
= & \frac{1}{2} \int_{-\infty}^{\infty} H(\delta(x-\xi)) d\{f(\xi)-g(\xi)\} \\
= & \int_{-\infty}^{\infty}\left(\int_{u}^{x} J_{\delta}(\omega-\xi) d \omega\right) d\{f(\xi)-g(\xi)\} \\
& +\frac{1}{2} \int_{-\infty}^{\infty} H(\delta(u-\xi)) d\{f(\xi)-g(\xi)\}
\end{aligned}
$$


Here $u$ is an arbitrary real number less than $x$. Applying the Fourier inversion formula to $J$, we obtain

$$
\begin{aligned}
\int_{u}^{x} J_{\delta}(\omega-\xi) d \omega & =\int_{u}^{x} \int_{-\delta}^{\delta} \hat{J}_{\delta}(t) e((\omega-\xi) t) d t d \omega \\
& =\int_{-\delta}^{\delta} \hat{J}_{\delta}(t)\left\{\int_{u}^{x} e(\omega t) d \omega\right\} e(-\xi t) d t
\end{aligned}
$$

Using Fubini's theorem we find that

$$
\begin{aligned}
& \int_{-\infty}^{\infty}\left(\int_{u}^{x} J_{\delta}(\omega-\xi) d \omega\right) d\{f(\xi)-g(\xi)\} \\
& \quad=\int_{-\delta}^{\delta} \hat{J}_{\delta}(t)\left\{\int_{u}^{x} e(\omega t) d \omega\right\}\left\{\int_{-\infty}^{\infty} e(-\xi t) d\{f(\xi)-g(\xi)\}\right\} d t \\
& \quad=\int_{-\delta}^{\delta} \hat{J}_{\delta}(t)\{e(x t)-e(u t)\}(2 \pi i t)^{-1}\{\widehat{d f}(t)-\widehat{d g}(t)\} d t
\end{aligned}
$$

Since the function $(2 \pi i t)^{-1}\{\widehat{d f}(t)-\widehat{d g}(t)\}$ is integrable on $[-\delta, \delta]$, we may combine (5.4) and (5.6) in the form

$$
\begin{aligned}
(f-g) * J_{\delta}(x)= & \int_{-\delta}^{\delta} \hat{J}_{\delta}(t)(2 \pi i t)^{-1}\{\widehat{d f}(t)-\widehat{d g}(t)\} e(x t) d t \\
& -\int_{-\delta}^{\delta} \hat{J}_{\delta}(t)(2 \pi i t)^{-1}\{\widehat{d f}(t)-\widehat{d g}(t)\} e(u t) d t \\
& +\frac{1}{2} \int_{-\infty}^{\infty} H(\delta(u-\xi)) d\{f(\xi)-g(\xi)\}
\end{aligned}
$$

We now let $u \rightarrow-\infty$. The second integral on the right of (5.7) tends to zero by the Riemann-Lebesgue lemma. In the third integral on the right of (5.7) we use (2.24) and the dominated convergence theorem. We obtain

$$
\lim _{u \rightarrow-\infty} \frac{1}{2} \int_{-\infty}^{\infty} H(\delta(u-\xi)) d\{f(\xi)-g(\xi)\}=-\frac{1}{2} \int_{-\infty}^{\infty} d\{f(\xi)-g(\xi)\}=0
$$

so

$$
(f-g) * J_{\delta}(x)=\int_{-\delta}^{\delta} \hat{J}_{\delta}(t)(2 \pi i t)^{-1}\{\widehat{d f}(t)-\widehat{d g}(t)\} e(x t) d t
$$

Next we write the second term on the right of (5.3) as

$$
\begin{aligned}
\frac{1}{2 \delta} \int_{-\infty}^{\infty} K_{\delta}(x-\xi) d\{f(\xi)-g(\xi)\} \\
=\frac{1}{2 \delta} \int_{-\delta}^{\delta} \hat{K}_{\delta}(t)\left\{\int_{-\infty}^{\infty} e((x-\xi) t) d\{f(\xi)-g(\xi)\}\right\} d t \\
=\frac{1}{2 \delta} \int_{-\delta}^{\delta} \hat{K}_{\delta}(t)\{\widehat{d f}(t)-\widehat{d g}(t)\} e(x t) d t
\end{aligned}
$$


Finally, the last three terms on the right of (5.3) can be estimated using (1.3), (4.3), and our bound on the density function $g^{\prime}(x)$. We find that

$$
\begin{aligned}
g * J_{\delta} & (x)+(2 \delta)^{-1}(d g) * K_{\delta}(x)-g(x) \\
& =\frac{1}{2} \int_{-\infty}^{\infty} B(\delta(x-\xi))-\operatorname{sgn}(\delta(x-\xi)) d g(\xi) \\
& \leqslant \frac{1}{2} M \int_{-\infty}^{\infty} B(\delta(x-\xi))-\operatorname{sgn}(\delta(x-\xi)) d \xi \\
& =(2 \delta)^{-1} M .
\end{aligned}
$$

When we combine (5.8)-(5.10) we obtain an upper bound for $f(x)-g(x)$. Of course, (4.2) also provides a minorizing inequality

$$
f(x) \geqslant f * J_{\delta}(x)-(2 \delta)^{-1}(d f) * K_{\delta}(x) .
$$

This leads to a corresponding lower bound for $f(x)-g(x)$. To complete the proof, the upper and lower bounds can be written together as (5.2).

By making a slight variation in our proof of Theorem 13 we get a similar inequality, but without the requirement that $g(x)$ have a bounded density.

THEOREM 14. Let $f(x)$ and $g(x)$ be probability distribution functions such that $t^{-1}\{\widehat{d f}(t)-\widehat{d g}(t)\}$ is integrable on a neighborhood of zero. Then

$$
\begin{gathered}
\left|f(x)-g(x)-\int_{-\delta}^{\delta} \hat{J}\left(\delta^{-1} t\right)(2 \pi i t)^{-1}\{\widehat{d f}(t)-\widehat{d g}(t)\} e(x t) d t\right| \\
\leqslant \frac{1}{2 \delta} \int_{-\delta}^{\delta} \hat{K}\left(\delta^{-1} t\right)\{\widehat{d f}(t)+\widehat{d g}(t)\} e(x t) d t
\end{gathered}
$$

for all real $x$ and all $\delta>0$.

Proof. We apply (4.2), instead of (5.3), directly to the function $f(x)-g(x)$. Since $f$ and $g$ are increasing and $K_{\delta}(x)$ is nonnegative, we have

$$
\begin{aligned}
\left|f(x)-g(x)-(f-g) * J_{\delta}(x)\right| & \leqslant(2 \delta)^{-1}\left(d V_{f-g}\right) * K_{\delta}(x) \\
& \leqslant(2 \delta)^{-1}(d f+d g) * K_{\delta}(x) .
\end{aligned}
$$

The result now follows by using (5.8) and (5.9) as in our proof of Theorem 13.

6. Further applications. Let $\lambda_{1}, \lambda_{2}, \ldots, \lambda_{N}$ be real numbers and

$$
f(x)=\sum_{n=1}^{N} a(n) e\left(\lambda_{n} x\right)
$$

an almost periodic trigonometric polynomial.

TheOREM 15. If $\left|\lambda_{n}\right| \geqslant \delta>0$ for $n=1,2, \ldots, N$, then

$$
\sup _{x}|f(x)| \leqslant(4 \delta)^{-1} \sup _{\xi}\left|f^{\prime}(\xi)\right| \text {. }
$$


If , in addition, $f(x)$ is real valued, then

$$
\sup _{x}|f(x)| \leqslant(2 \delta)^{-1} \sup _{\xi} f^{\prime}(\xi) .
$$

Moreover, the constants $(4 \delta)^{-1}$ and $(2 \delta)^{-1}$ are asymptotically best possible as $N \rightarrow \infty$.

Proof. We have

$$
\begin{aligned}
f * I_{2 \delta}(x) & =\int_{-\infty}^{\infty} f(x-\xi) I_{2 \delta}(\xi) d \xi \\
& =\sum_{n=1}^{N} a(n) e\left(\lambda_{n} x\right) \int_{-\infty}^{\infty} I_{2 \delta}(\xi) e\left(-\lambda_{n} \xi\right) d \xi \\
& =\sum_{n=1}^{N} a(n) \hat{I}_{2 \delta}\left(\lambda_{n}\right) e\left(\lambda_{n} x\right)=0,
\end{aligned}
$$

since $\hat{I}_{2 \delta}(t)=0$ for $|t| \geqslant \delta$. It follows that

$$
\begin{aligned}
f(x) & =\frac{1}{2} \int_{-\infty}^{\infty} f(x-\xi) d\{\operatorname{sgn}(2 \delta \xi)\} \\
& =\frac{1}{2} \int_{-\infty}^{\infty} f(x-\xi) d\{\operatorname{sgn}(2 \delta \xi)-G(2 \delta \xi)\} \\
& =\frac{1}{2} \int_{-\infty}^{\infty}\{\operatorname{sgn}(2 \delta \xi)-G(2 \delta \xi)\} f^{\prime}(x-\xi) d \xi .
\end{aligned}
$$

Therefore, by Theorem 4 ,

$$
\begin{aligned}
|f(x)| & \leqslant\left(\sup _{\xi}\left|f^{\prime}(\xi)\right|\right)\left(\frac{1}{2} \int_{-\infty}^{\infty}|\operatorname{sgn}(2 \delta u)-G(2 \delta u)| d u\right) \\
& =(4 \delta)^{-1} \sup _{\xi}\left|f^{\prime}(\xi)\right| .
\end{aligned}
$$

This proves (6.1).

Since $\hat{J}_{\delta}(t)=\hat{K}_{\delta}(t)=0$ for $|t| \geqslant \delta$, we also have

$$
f * J_{\delta}(x)=\delta^{-1} f^{\prime} * K_{\delta}(x)=0
$$

for all real $x$. This implies that

$$
\begin{aligned}
f(x) & =\frac{1}{2} \int_{-\infty}^{\infty}\{\operatorname{sgn}(\delta \xi)-H(\delta \xi)\} f^{\prime}(x-\xi) d \xi \\
& =\frac{1}{2} \int_{-\infty}^{\infty}\{\operatorname{sgn}(\delta \xi)-H(\delta \xi) \pm K(\delta \xi)\} f^{\prime}(x-\xi) d \xi .
\end{aligned}
$$

Now we suppose that $f(x)$ is real valued and use the minus sign on the right of (6.3). In view of the bound (2.24) we find that

$$
\text { (6.4) } \begin{aligned}
f(x) & \geqslant\left(\sup _{\xi} f^{\prime}(\xi)\right)\left(\frac{1}{2} \int_{-\infty}^{\infty}\{\operatorname{sgn}(\delta u)-H(\delta u)-K(\delta u)\} d u\right) \\
& =-(2 \delta)^{-1} \sup _{\xi} f^{\prime}(\xi) .
\end{aligned}
$$


Similarly, using the plus sign on the right of (6.3), we obtain

$$
f(x) \leqslant(2 \delta)^{-1} \sup _{\xi} f^{\prime}(\xi)
$$

for all real $x$. This establishes (6.2).

In order to show that (6.1) and (6.2) are sharp, let

$$
k_{N}(x)=\sum_{n=-N}^{N}\left(1-\frac{|n|}{N+1}\right) e(n x)=(N+1)^{-1}\left(\frac{\sin \pi(N+1) x}{\sin \pi x}\right)^{2}
$$

denote the periodic Fejer kernel. Then let

$$
\psi(x)= \begin{cases}x-[x]-1 / 2 & \text { if } x \notin \mathbf{Z}, \\ 0 & \text { if } x \in \mathbf{Z} .\end{cases}
$$

It suffices to assume that $\delta=1$; then the function

$$
\begin{aligned}
f_{N}(x) & =\psi * k_{N}(x)=\int_{0}^{1} \psi(\xi) k_{N}(x-\xi) d \xi \\
& =-\sum_{\substack{n=-N \\
n \neq 0}}^{N}(2 \pi i n)^{-1}\left(1-\frac{|n|}{N+1}\right) e(n x)
\end{aligned}
$$

is real valued and satisfies the hypotheses of the theorem. We have

$$
f_{N}^{\prime}(x)=-\sum_{\substack{n=-N \\ n \neq 0}}^{N}\left(1-\frac{|n|}{N+1}\right) e(n x)=1-k_{N}(x)
$$

and hence the right side of $(6.2)$ is

$$
\frac{1}{2} \sup _{\xi} f_{N}^{\prime}(\xi)=\frac{1}{2}
$$

Since $f_{N}(x) \rightarrow \psi(x)$ pointwise, we must also have

$$
\lim _{N \rightarrow \infty}\left(\sup _{x}\left|f_{N}(x)\right|\right)=\frac{1}{2} \text {. }
$$

The proof that (6.1) is sharp is essentially the same, except $\|x\|-1 / 4$ is used in place of $\psi(x)$.

Inequality (6.1) is due to H. Bohr (see [Sha, p. 142]), and (6.2) was discovered by Beurling. In fact, this inequality motivated Beurling's construction of the extremal function $B(z)=H(z)+K(z)$. Theorem 15 can be extended to absolutely continuous functions $f(x)$ such that $f$ and $f^{\prime}$ are in $L^{\infty}(\mathbf{R})$ and the spectrum of $f$ does not intersect the open interval $(-2 \pi \delta, 2 \pi \delta)$. This extension for (6.1) is discussed by Shapiro [Sha, Chapter 7].

Next we consider a general form of Hilbert's inequality first obtained by Montgomery and Vaughan [M-V] (see also [Mon] and $\left[\mathbf{G V}_{2}\right]$ ). Here we give a new and particularly simple proof of this result.

Theorem 16 (Montgomery AND VAUghan). Let $\lambda_{1}, \lambda_{2}, \ldots, \lambda_{N}$ be real numbers satisfying $\left|\lambda_{m}-\lambda_{n}\right| \geqslant \delta>0$ whenever $m \neq n$, and let $a(1), \ldots, a(N)$ 
be arbitrary complex numbers. Then

$$
\left|\sum_{\substack{m=1 \\ m \neq n}}^{N} \sum_{\substack{n=1 \\ m}}^{N} \frac{a(m) \overline{a(n)}}{\lambda_{m}-\lambda_{n}}\right| \leqslant \pi \delta^{-1} \sum_{n=1}^{N}|a(n)|^{2} .
$$

Proof. Let $\varphi(x)=H(x)+K(x)-\operatorname{sgn}(x)$ so that $\varphi(x)$ is nonnegative and integrable. Using (2.29) and (2.34) we see that

$$
\hat{\varphi}(t)= \begin{cases}1 & \text { if } t=0 \\ -(\pi i t)^{-1} & \text { if }|t| \geqslant 1\end{cases}
$$

It follows that

$$
\begin{aligned}
0 & \leqslant \int_{-\infty}^{\infty} \varphi_{\delta}(x)\left|\sum_{m=1}^{N} a(m) e\left(-\lambda_{m} x\right)\right|^{2} d x \\
& =\sum_{m=1}^{N} \sum_{n=1}^{N} a(m) \overline{a(n)} \hat{\varphi}_{\delta}\left(\lambda_{m}-\lambda_{n}\right) \\
& =\sum_{n=1}^{N}|a(n)|^{2} \hat{\varphi}_{\delta}(0)-\sum_{\substack{m=1 \\
m \neq n}}^{N} \sum_{\substack{n=1 \\
m \neq n}}^{N} a(m) \overline{a(n)}\left\{\pi i \delta^{-1}\left(\lambda_{m}-\lambda_{n}\right)\right\}^{-1},
\end{aligned}
$$

and therefore

$$
\sum_{\substack{m=1 \\ m \neq n}}^{N} \sum_{\substack{n=1 \\ i\left(\lambda_{m}-\lambda_{n}\right)}}^{N} \leqslant \pi \delta^{-1} \sum_{n=1}^{N}|a(n)|^{2} .
$$

If we begin with $H(x)-K(x)-\operatorname{sgn}(x)$, we obtain (6.8) with the inequality reversed and a factor of -1 on the right side. These bounds prove the theorem.

7. Majorizing periodic functions. We define periodic trigonometric polynomials $i_{N}(x), j_{N}(x)$, and $k_{N}(x)$ as follows:

$$
\begin{aligned}
& i_{N}(x)=\sum_{m=-\infty}^{\infty} I_{2 N+2}(x+m)=\sum_{n=-N}^{N} \hat{I}_{2 N+2}(n) e(n x), \\
& j_{N}(x)=\sum_{m=-\infty}^{\infty} J_{N+1}(x+m)=\sum_{n=-N}^{N} \hat{J}_{N+1}(n) e(n x), \\
& k_{N}(x)=\sum_{m=-\infty}^{\infty} K_{N+1}(x+m)=\sum_{n=-N}^{N} \hat{K}_{N+1}(n) e(n x) .
\end{aligned}
$$

Of course, $k_{N}$ is the periodic Fejer kernel also given by (6.5). The identities indicated on the right of (7.1)-(7.3) follow immediately from the Poisson summation formula. For the purpose of approximating or majorizing periodic functions by trigonometric polynomials, we use $i_{N}, j_{N}$, and $k_{N}$ in a role analogous to that played by the entire functions $I, J$, and $K$ in Theorem 11 . In the periodic case $\psi(x)$, defined by (6.6), can be used in place of $\operatorname{sgn}(x)$. Thus, 
we assume throughout this section that $\psi$ is so defined. Here we use $f * g(x)$ to denote the convolution

$$
f * g(x)=\int_{-1 / 2}^{1 / 2} f(x-\xi) g(\xi) d \xi
$$

of two periodic functions with period 1 . We also write

$$
\hat{f}(n)=\int_{-1 / 2}^{1 / 2} f(x) e(-n x) d x
$$

for the $n$th Fourier coefficient of the periodic function $f(x)$.

THEOREM 17. The trigonometric polynomial

$$
\psi * i_{N}(x)=\sum_{\substack{n=-N \\ n \neq 0}}^{N}(-2 \pi i n)^{-1} \hat{I}_{2 N+2}(n) e(n x)
$$

satisfies

$$
\begin{gathered}
\operatorname{sgn}\left(\psi * i_{N}(x)\right)=\operatorname{sgn}(\psi(x)), \\
\operatorname{sgn}\left(\psi * i_{N}(x)-\psi(x)\right)=\operatorname{sgn}(\sin 2 \pi(N+1) x),
\end{gathered}
$$

and

$$
\int_{-1 / 2}^{1 / 2}\left|\psi * i_{N}(x)-\psi(x)\right| d x=(4 N+4)^{-1}
$$

If $p_{N}(x)$ is any trigonometric polynomial of degree at most $N$, then

$$
\int_{-1 / 2}^{1 / 2}\left|p_{N}(x)-\psi(x)\right| d x \geqslant(4 N+4)^{-1}
$$

Moreover, there is equality in (7.8) if and only if $p_{N}(x)=\psi * i_{N}(x)$.

Proof. We have

$$
\begin{aligned}
\psi * i_{N}(x) & =-\sum_{n=1}^{N} \hat{I}_{2 N+2}(n)\left(\frac{\sin 2 \pi n x}{\pi n}\right) \\
& =-\sum_{n=1}^{N}\left\{\hat{I}_{2 N+2}(n)-\hat{I}_{2 N+2}(n+1)\right\} S(x, n),
\end{aligned}
$$

where

$$
S(x, n)=\sum_{l=1}^{n} \frac{\sin 2 \pi l x}{\pi l} .
$$

By a classical result of Fejer [Fej, Satz XXVI] the partial sums $S(x, n)$ are positive for $0<x<1 / 2$. Since $\hat{I}_{2 N+2}(n)$ is strictly decreasing for $n=$ $1,2, \ldots, N+1$ (by (2.10)), the right side of (7.9) is negative for $0<x<1 / 2$. As $\psi * i_{N}(x)$ is odd, continuous, and periodic, this proves (7.5) for all real $x$.

Let $D(x)$ be defined as in Corollary 3 and write $\delta=2 N+2$. Then

$$
\begin{aligned}
-\frac{1}{2 \delta} & \sum_{m=-\infty}^{\infty} D_{\delta}(x+m)=-\frac{1}{2 \delta} \sum_{n=-\infty}^{\infty} \hat{D}_{\delta}(n) e(n x) \\
& =\sum_{\substack{n=-\infty \\
n \neq 0}}^{\infty}-\frac{1}{2 \pi i n}\left\{\hat{I}_{2 N+2}(n)-1\right\} e(n x)=\psi * i_{N}(x)-\psi(x)
\end{aligned}
$$


by (2.15) and the Poisson summation formula. Using (2.4) we have

$$
\begin{aligned}
\operatorname{sgn}\left\{-(2 \delta)^{-1} D_{\delta}(x+m)\right\} & =\operatorname{sgn}\{\sin 2 \pi(N+1)(x+m)\} \\
& =\operatorname{sgn}\{\sin 2 \pi(N+1) x\}
\end{aligned}
$$

for all real $x$ and every integer $m$. This clearly implies (7.6).

To establish (7.7) we combine (2.16), (7.10), and (7.11). We find that

$$
\begin{aligned}
& \int_{-1 / 2}^{1 / 2}\left|\psi * i_{N}(x)-\psi(x)\right| d x=\frac{1}{2 \delta} \int_{-1 / 2}^{1 / 2}\left|\sum_{m=-\infty}^{\infty} D_{\delta}(x+m)\right| d x \\
& =\frac{1}{2 \delta} \sum_{m=-\infty}^{\infty} \int_{-1 / 2}^{1 / 2}\left|D_{\delta}(x+m)\right| d x=\frac{1}{2 \delta} \int_{-\infty}^{\infty}\left|D_{\delta}(x)\right| d x=(4 N+4)^{-1}
\end{aligned}
$$

To obtain (7.8) we proceed in a manner quite similar to our proof of (2.16). From the Fourier expansion (2.20) we have

$$
\int_{-1 / 2}^{1 / 2} p_{N}(x) \operatorname{sgn}\{\sin 2 \pi(N+1) x\} d x=0
$$

for every trigonometric polynomial $p_{N}(x)$ having degree at most $N$. This leads to the lower bound

$$
\begin{aligned}
\int_{-1 / 2}^{1 / 2} \mid & p_{N}(x)-\psi(x) \mid d x \\
& \geqslant\left|\int_{-1 / 2}^{1 / 2}\left\{p_{N}(x)-\psi(x)\right\} \operatorname{sgn}\{\sin 2 \pi(N+1) x\} d x\right| \\
& =\left|\int_{-1 / 2}^{1 / 2} \psi(x) \operatorname{sgn}\{\sin 2 \pi(N+1) x\} d x\right| \\
& =\left|\frac{2}{\pi i} \sum_{k=-\infty}^{\infty}(2 k+1)^{-1} \int_{-1 / 2}^{1 / 2} \psi(x) e\{(2 k+1)(N+1) x\} d x\right| \\
& =\pi^{-2}(N+1)^{-1} \sum_{k=-\infty}^{\infty}(2 k+1)^{-2}=(4 N+4)^{-1}
\end{aligned}
$$

If there is equality in (7.12) then $p_{N}(x)$ interpolates the values of $\psi(x)$ at the points $l(2 N+2)^{-1}$ for $l=1,2, \ldots,(2 N+1)$. Since the degree of $p_{N}$ is at most $N$, such an interpolating polynomial exists and is unique [Zyg, Vol. II, pp. $1-3$ ]. In view of (7.6) the unique interpolating polynomial is precisely $\psi * i_{N}(x)$.

The determination of a unique extremal trigonometric polynomial in inequality (7.8) is a basic step in the sharp forms of Jackson's theorems obtained by Favard [Fav] and Achieser and Krein [A-K] (see also Cheney [Che, pp. 139-148]). A typical approximation theorem of this type is given below as (7.23).

Our next result identifies an extremal property associated with $\psi * j_{N}(x)+$ $(2 N+2)^{-1} k_{N}(x)$. 
THEOREM 18. The trigonometric polynomial

satisfies

$$
\psi * j_{N}(x)=\sum_{\substack{n=-N \\ n \neq 0}}^{N}(-2 \pi i n)^{-1} \hat{J}_{N+1}(n) e(n x)
$$

$$
\begin{gathered}
\operatorname{sgn}\left(\psi * j_{N}(x)\right)=\operatorname{sgn}(\psi(x)) \\
\left|\psi * j_{N}(x)-\psi(x)\right| \leqslant(2 N+2)^{-1} k_{N}(x),
\end{gathered}
$$

and

$$
\left|\psi * j_{N}(x)\right| \leqslant|\psi(x)|
$$

If $p_{N}(x)$ is any trigonometric polynomial of degree at most $N$ satisfying $p_{N}(x) \geqslant$ $\psi(x)$ for all real $x$, then

$$
\int_{-1 / 2}^{1 / 2} p_{N}(x)-\psi(x) d x \geqslant(2 N+2)^{-1}
$$

Moreover, there is equality in (7.16) if and only if

$$
p_{N}(x)=\psi * j_{N}(x)+(2 N+1)^{-1} k_{N}(x) .
$$

Proof. Identity (7.13) is established in the same way as (7.5).

Next we let $E(x)$ be defined as in Corollary 7. Mimicking our proof of (7.10) we find that

$$
(2 N+2)^{-1} \sum_{m=-\infty}^{\infty} E_{N+1}(x+m)=\psi * j_{N}(x)-\psi(x)
$$

Hence,

$$
\left|\psi * j_{N}(x)-\psi(x)\right| \leqslant(2 N+2)^{-1} \sum_{m=-\infty}^{\infty} K_{N+1}(x+m)=(2 N+2)^{-1} k_{N}(x)
$$

by combining (2.24), (7.3), and (7.17).

Inequality (7.15) is somewhat more obscure. We use the identity (2.26) with $x>0$. A simple calculation shows that

$$
\begin{aligned}
-E(x) & =1-H(x)=\left(\frac{\sin \pi x}{\pi}\right)^{2}\left\{-2 x^{-1}+x^{-2}+2 \sum_{m=1}^{\infty}(x+m)^{-2}\right\} \\
& =\left(\frac{\sin \pi x}{\pi}\right)^{2}\left\{\int_{0}^{\infty}\left(-2+u+2 \sum_{m=1}^{\infty} u e^{-u m}\right) e^{-u x} d u\right\} \\
& =\left(\frac{\sin \pi x}{\pi}\right)^{2}\left\{4 \int_{0}^{\infty}(v \operatorname{coth} v-1) e^{-2 v x} d v\right\} .
\end{aligned}
$$

Since $v \operatorname{coth} v>1$ for $v>0$ we see that

$$
-E(x)=((\sin \pi x) / \pi)^{2} R(x)
$$


for $x>0$, where $R(x)$ is a positive, strictly decreasing function of $x$. Now we use (7.17), (7.18), and the fact that $E(x)$ is an odd function. It follows that

$$
\begin{aligned}
\psi * j_{N}(x)-\psi(x) & \\
= & -(2 N+2)^{-1}\left\{\sum_{m=0}^{\infty} E_{N+1}(x+m)-\sum_{m=0}^{\infty} E_{N+1}((1-x)+m)\right\} \\
= & (2 N+2)^{-1}\left(\frac{\sin \pi(N+1) x}{\pi}\right)^{2} \\
& \cdot \sum_{m=0}^{\infty}\left\{R_{N+1}(x+m)-R_{N+1}((1-x)+m)\right\} .
\end{aligned}
$$

From our observations concerning $R(x)$, the right side of (7.19) is clearly positive for $0<x<1 / 2$. This, together with (7.13), shows that (7.15) holds for $0<x<1 / 2$. Then the inequality holds for all real $x$, since both sides of (7.15) are even functions of period 1 .

Finally, we suppose that $p_{N}(x)$ has degree at most $N$ and majorizes $\psi(x)$. By continuity we must have $p_{N}(0) \geqslant 1 / 2$. Thus,

$$
\begin{aligned}
\frac{1}{2} & \leqslant \sum_{l=0}^{N}\left\{p_{N}\left(l(N+1)^{-1}\right)-\psi\left(l(N+1)^{-1}\right)\right\} \\
& =\sum_{n=-N}^{N} \hat{p}_{N}(n) \sum_{l=0}^{N} e\left(\ln (N+1)^{-1}\right)-\sum_{l=1}^{N}\left\{l(N+1)^{-1}-\frac{1}{2}\right\} \\
& =(N+1) \hat{p}_{N}(0) .
\end{aligned}
$$

As $\hat{\psi}(0)=0,(7.20)$ is equivalent to (7.16). If (7.20) holds with equality then

$$
p_{N}(0)=1 / 2 \text { and } p_{N}\left(l(N+1)^{-1}\right)=l(N+1)^{-1}-1 / 2
$$

for $l=1,2, \ldots, N$. Since $p_{N}(x)$ also majorizes $\psi(x)$, we must have

$$
p_{N}^{\prime}\left(l(N+1)^{-1}\right)=1
$$

for $l=1,2, \ldots, N$. It follows (see Zygmund [Zyg, Vol. II, p. 23]) that the $2 N+1$ conditions (7.21) and (7.22) determine a unique polynomial of degree at most $N$. From (7.13)-(7.15) we see that these conditions are also satisfied by $\psi * j_{N}(x)+(2 N+2)^{-1} k_{N}(x)$. This completes our proof of the theorem.

We now state a result which is the periodic analogue of Theorem 11. Here we suppose that $f: \mathbf{R} \rightarrow \mathbf{C}$ has period 1 and bounded variation on each closed interval of length 1 . We also assume that $f$ satisfies the normalizing condition (1.16). The total variation of $f$ on $\left[-\frac{1}{2}, x\right]$ will be denoted by $V_{f}(x)$, and $V_{f}=V_{f}\left(\frac{1}{2}\right)$. We write $\left(d V_{f}\right) * k_{N}(x)$ for the convolution

$$
\left(d V_{f}\right) * k_{N}(x)=\int_{-1 / 2}^{1 / 2} k_{N}(x-\xi) d V_{f}(\xi)
$$

THEOREM 19. The trigonometric polynomials $f * i_{N}(x), f * j_{N}(x)$, and $\left(d V_{f}\right) * k_{N}(x)$ have degree at most $N$ and satisfy

$$
\int_{-1 / 2}^{1 / 2}\left|f(x)-f * i_{N}(x)\right| d x \leqslant(4 N+4)^{-1} V_{f}
$$


and

$$
\left|f(x)-f * j_{N}(x)\right| \leqslant(2 N+2)^{-1}\left(d V_{f}\right) * k_{N}(x) .
$$

In particular, if $f$ is real valued, then

$$
m(f, N, x)=f * j_{N}(x)+(2 N+2)^{-1}\left(d V_{f}\right) * k_{N}(x)
$$

has degree at most $N$, satisfies

$$
f(x) \leqslant m(f, N, x),
$$

and

$$
\int_{-1 / 2}^{1 / 2} m(f, N, x)-f(x) d x=(2 N+2)^{-1} V_{f} .
$$

Proof. From (7.4) we have

$$
(d / d x) \psi * i_{N}(x)=1-i_{N}(x) .
$$

This can be used to verify the identity

$$
\begin{aligned}
\int_{-1 / 2}^{1 / 2} f( & x-\xi) d\left\{\psi * i_{N}(\xi)-\psi(\xi)\right\} \\
\quad & \int_{-1 / 2}^{1 / 2} f(x-\xi)\left\{1-i_{N}(\xi)\right\} d \xi-\int_{-1 / 2}^{1 / 2} f(x-\xi) d \psi(\xi) \\
& =f(x)-f * i_{N}(x)
\end{aligned}
$$

at all continuity points $x$ of $f$. Integrating the left side of (7.28) by parts, we find that

$$
\int_{-1 / 2}^{1 / 2} \psi * i_{N}(x-\xi)-\psi(x-\xi) d f(\xi)=f(x)-f * i_{N}(x)
$$

at all continuity points $x$. Since $f$ is continuous almost everywhere,

$$
\begin{aligned}
& \int_{-1 / 2}^{1 / 2}\left|f(x)-f * i_{N}(x)\right| d x \\
& \quad \leqslant \int_{-1 / 2}^{1 / 2} \int_{-1 / 2}^{1 / 2}\left|\psi * i_{N}(x-\xi)-\psi(x-\xi)\right| d x d V_{f}(\xi) \\
& \quad=(4 N+4)^{-1} V_{f} .
\end{aligned}
$$

Similarly, we deduce (7.29), but with $i_{N}$ replaced by $j_{N}$. Hence, at each continuity point $x$ of $f$ we have

$$
\text { (7.30) } \begin{aligned}
\left|f(x)-f * j_{N}(x)\right| & \leqslant \int_{-1 / 2}^{1 / 2}\left|\psi * j_{N}(x-\xi)-\psi(x-\xi)\right| d V_{f}(\xi) \\
& \leqslant(2 N+2)^{-1} \int_{-1 / 2}^{1 / 2} k_{N}(x-\xi) d V_{f}(\xi) \\
& =(2 N+2)^{-1}\left(d V_{f}\right) * k_{N}(x)
\end{aligned}
$$

Using the normalization (1.9) we find that (7.24) holds for all real $x$. Of course, (7.26) is an immediate consequence of (7.30). 
8. The Erdös-Turán inequality. Let $E$ denote an interval of $\mathbf{R}$ having length $|E|<1$ and let $\chi_{E}(x)$ be the normalized characteristic function of $E$. Throughout this section $\sigma_{E}(x)$ will denote the periodic function

$$
\sigma_{E}(x)=\left\{\sum_{l=-\infty}^{\infty} \chi_{E}(x+l)\right\}-|E|
$$

We also suppose that $\left\{\xi_{1}, \xi_{2}, \ldots\right\}$ is a sequence of real numbers, and we write $\vec{\xi}_{M}=\left\{\xi_{1}, \xi_{2}, \ldots, \xi_{M}\right\}$ for the first $M$ terms. As is well known, the sequence $\left\{\xi_{1}, \xi_{2}, \ldots\right\}$ is said to be uniformly distributed $\bmod 1$ if

$$
\lim _{M \rightarrow \infty} M^{-1} \sum_{m=1}^{M} \sigma_{E}\left(\xi_{m}\right)=0
$$

for every interval $E$. By Weyl's criterion [K-N, p. 7] this is equivalent to the requirement that

$$
\lim _{M \rightarrow \infty} M^{-1} \sum_{m=1}^{M} e\left(l \xi_{m}\right)=0
$$

for every positive integer $l$.

For each positive integer $M$ we define the discrepancy $\Delta\left(\vec{\xi}_{M}\right)$ of $\vec{\xi}_{M}$ by

$$
\Delta\left(\vec{\xi}_{M}\right)=\sup _{E} M^{-1}\left|\sum_{m=1}^{M} \sigma_{E}\left(\xi_{m}\right)\right| .
$$

Then another form of Weyl's criterion states that the sequence $\left\{\xi_{1}, \xi_{2}, \ldots\right\}$ is uniformly distributed mod 1 if and only if $\lim _{M \rightarrow \infty} \Delta\left(\vec{\xi}_{M}\right)=0$. An important inequality of Erdös and Turán [E-T] provides an upper bound for the discrepancy $\Delta\left(\vec{\xi}_{M}\right)$ in terms of the sums $M^{-1} \sum_{m=1}^{M} e\left(l \xi_{m}\right)$ for $l$ in a finite range (see also [N-P] and [K-N, p. 112]). Our ability to majorize $\sigma_{E}(x)$ by a trigonometric polynomial is ideally suited to proving results of this type. In fact, we could also use (8.1) and Selberg's function (1.5), which majorizes $\chi_{E}(x)$. This application, as well as our Corollary 21 , was first observed by $\mathrm{H}$. L. Montgomery.

THEOREM 20. For each interval $E$ of length $|E|<1$ and every integer $N \geqslant 1$ we have

$$
\begin{aligned}
\left|\sum_{m=1}^{M} \sigma_{E}\left(\xi_{m}\right)\right| \leqslant & (N+1)^{-1}\left\{M+2 \sum_{n=1}^{N} \hat{K}_{N+1}(n)\left|\sum_{m=1}^{M} e\left(n \xi_{m}\right)\right|\right\} \\
& +2 \sum_{n=1}^{N} \frac{|\sin \pi n| E||}{\pi n} \hat{J}_{N+1}(n)\left|\sum_{m=1}^{M} e\left(n \xi_{m}\right)\right| .
\end{aligned}
$$


Proof. If $f(x)$ satisfies the hypotheses of Theorem 19 and $\hat{f}(0)=0$, then

$$
\begin{aligned}
\left|\sum_{m=1}^{M} f\left(\xi_{m}\right)\right| \leqslant & (2 N+2)^{-1} \sum_{m=1}^{M}\left(d V_{f}\right) * k_{N}\left(\xi_{m}\right)+\left|\sum_{m=1}^{M} f * j_{N}\left(\xi_{m}\right)\right| \\
\leqslant & (2 N+2)^{-1} V_{f} \sum_{n=-N}^{N} \hat{K}_{N+1}(n)\left|\sum_{m=1}^{M} e\left(n \xi_{m}\right)\right| \\
& +\sum_{\substack{n=-N \\
n \neq 0}}^{N}|\hat{f}(n)| \hat{J}_{N+1}(n)\left|\sum_{m=1}^{M} e\left(n \xi_{m}\right)\right| .
\end{aligned}
$$

Now we let $f(x)=\sigma_{E}(x)$. It follows that $V_{f}=2$ and

$$
|\hat{f}(n)|=(\pi n)^{-1}|\sin \pi n| E||
$$

for $n \neq 0$. Hence, inequality (8.4) implies (8.3).

If we ignore the factors $\hat{K}_{N+1}$ and $\hat{J}_{N+1}$ on the right of (8.3), we obtain a bound on the discrepancy of the form

$$
\Delta\left(\vec{\xi}_{M}\right) \leqslant(N+1)^{-1}+2 \sum_{n=1}^{N}\left\{(\pi n)^{-1}+(N+1)^{-1}\right\}\left|M^{-1} \sum_{m=1}^{M} e\left(n \xi_{m}\right)\right|
$$

Alternatively, if the length $|E|$ is small, the factor $|\sin \pi n| E \|$ can be used to advantage.

COROLlARY 21. Suppose that $\xi_{1}, \xi_{2}, \ldots, \xi_{M}$ are real numbers satisfying $\left\|\xi_{m}\right\|$ $\geqslant X^{-1}$ for some real parameter $X \geqslant 2$. Then

$$
M \leqslant 6 \sum_{1 \leqslant n \leqslant X}\left|\sum_{m=1}^{M} e\left(n \xi_{m}\right)\right|
$$

Proof. Let $E=\left(-X^{-1}, X^{-1}\right)$ and $N=[X]$. Since the right side of (8.5) is continuous in each $\xi_{m}$, we may suppose $\left\|\xi_{m}\right\|>X^{-1}$. Then

$$
\left|\sum_{m=1}^{M} \sigma_{E}\left(\xi_{m}\right)\right|=M|E|=2 M X^{-1}
$$

and, using $|\sin \pi n| E \| \leqslant \pi n|E|$, this is bounded from above by

$$
\begin{aligned}
(N+1)^{-1} M+2 \sum_{n=1}^{N}\left\{(N+1)^{-1}\right. & +|E|\}\left|\sum_{m=1}^{M} e\left(n \xi_{m}\right)\right| \\
& \leqslant M X^{-1}+6 X^{-1} \sum_{n=1}^{N}\left|\sum_{m=1}^{M} e\left(n \xi_{m}\right)\right|
\end{aligned}
$$

The corollary follows from (8.6) and (8.7).

We note that (8.5) has been used recently in the work of Baker and Harman [B-H]. 


\section{REFERENCES}

[A-K] N. I. Achieser and M. G. Krein, Best approximation of differentiable periodic functions by means of trigonometric sums, Dokl. Akad. Nauk SSSR 15 (1937), 107-112. (Russian)

[B-H] R. C. Baker and G. Harman, Small fractional parts of quadratic forms, Proc. Edinburgh Math. Soc. 25 (1982), 269-277.

[vBe] P. van Beek, An application of Fourier methods to the problem of sharpening the Berry-Esseen inequality, Z. Wahrsch. verw. Gebiete. 23 (1972), 187-196.

[Brn] S. N. Bernstein, Sur une propriété des fonctions entieres, C. R. Acad. Sci. 176 (1923), 1603-1605.

[Brr] A. C. Berry, The accuracy of the Gaussian approximation to the sum of independent varlates, Trans. Amer. Math. Soc. 49 (1941), 122-136.

[Beu] A. Beurling, Sur les intégrales de Fourier absolument convergentes et leur application à une transformation fonctionelle, Neuvième Congrès Math. Scandinaves, Helsingfors, 1938.

[Boa] R. P. Boas, Entire functions, Academic Press, New York, 1954.

[Che] E. W. Cheney, Introduction to approximation theory, Chelsea, New York, 1982.

[E-T] P. Erdös and P. Turán, On a problem in the theory of uniform distribution. I, Indag. Math. 10 (1948), 370-378.

[Ess] C. G. Esseen, On the Liapunov limit of error in the theory of probability, Ark. Mat.-Astr. Fys. 28A (1942), 1-19.

[Fav] J. Favard, Sur les meilleurs procédés d'approximation de certaines classes de fonctions par des polynômes trigonométriques, Bull. Sci. Math. 61 (1937), 209-224, 243-256.

[Fej] L. Fejer, Einige Sätze, die sich auf das Vorzeichen einer ganzen rationalen Function bezeihen nebst Anwendungen, Monatsh. Math. Phys. 35 (1928), 305-344.

[Fel] W. Feller, An introduction to probability theory and its applications, Vol. II, Wiley, New York, 1971.

$\left[\mathbf{G V}_{1}\right]$ S. W. Graham and J. D. Vaaler, Extremal functions for the Fourier transform and the large sieve, Topics in Classical Number Theory (G. Halász, ed.), North-Holland, New York, 1984.

$\left[\mathbf{G V}_{2}\right] \ldots$ A class of extremal functions for the Fourier transform, Trans. Amer. Math. Soc. 265 (1981), 283-302.

[K-N] L. Kuipers and H. Niederreiter, Uniform distribution of sequences, Wiley, New York, 1974.

[Loe] M. Loève, Probability theory, 3rd ed. van Nostrand, Princeton, N. J., 1963.

[Log] B. F. Logan, Bandlimited functions bounded below over an interval, Notices Amer. Math. Soc. 24 (1977), A-331.

[Mon] Hugh L. Montgomery, The analytic principle of the large sieve, Bull. Amer. Math. Soc. 84 (1978), 547-567.

[M-V] H. L. Montgomery and R. C. Vaughan, Hilbert's inequality, J. London Math. Soc. (2) 8 (1974), 73-81.

[N-P] H. Niederreiter and W. Philipp, Berry-Esseen bounds and a theorem of Erdös and Turán on uniform distribution mod 1, Duke Math. J. 40 (1973), 633-649.

[P-P] M. Plancherel and G. Polya, Fonctions entières et intégrales de Fourier multiples, (Seconde partie) Comment. Math. Helv. 10 (1938), 110-163.

[Pr. $\mathbf{P r}_{1}$ H. Prawitz, Limits for a distribution, if the characteristic function is given in a finite domain, Skand. Aktur Tidskr, (1972), 138-154.

$\left[\mathbf{P r}_{2}\right] \_$Ungleichungen für den absoluten Betrag einer charakteristischen Funktion, Skand. Akturatidskr (1973), 11-16.

[Pr $\left.\mathbf{P r}_{3}\right]$, Weiter Ungleichungen für den absoluten Betrag einer charakteristischen Funktion, Scand. Actuarial J. (1975), 21-28.

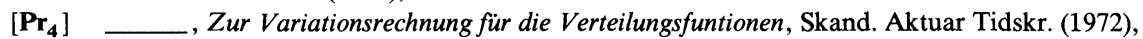
$202-208$.

[Pr $\left.\mathbf{P r}_{5}\right]$, On the remainder in the central limit theorem, Scand. Actuarial J. (1975), 145-156.

[Sel] A. Selberg, Remarks on sieves, Proc. 1972 Number Theory Conf. (Univ. of Colorado, Boulder), pp. 205-216.

[Sha] H. S. Shapiro, Topics in approximation theory, Lecture Notes in Math., vol. 187, SpringerVerlag, New York, 1971.

[SNa] B. Sz. Nagy, Über gewisse Extremalfragen bei transformierten trigonometrischen Entwicklungen. II, Ber. Math.-Phys. Kl. Sächs Akad. Wiss. Leipzig 91 (1939). 
[StW] E. M. Stein and G. Weiss, Introduction to Fourier analysis on Euclidean spaces, Princeton Univ. Press, Princeton, N. J., 1971.

[Tim] A. F. Timan, Theory of approximation of functions of a real variable, Pergamon Press-Macmillan, New York, (1963).

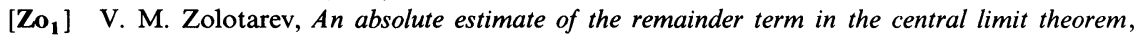
Theor. Probab. Appl. 11 (1966), 95-105.

$\left[\mathbf{Z o}_{2}\right]$ Some inequalities in probability theory and their application in sharpening the Lyapunov theorem, Soviet Math. Dokl. 8 (1967), 1427-1430.

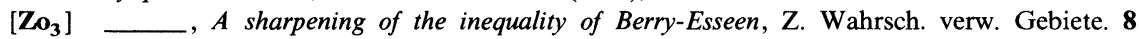
(1967), 332-342.

[Zyg] A. Zygmund, Trigonometric series, Vols. I, II, Cambridge Univ. Press, 1968.

Department of Mathematics, University of TeXas, Austin, TeXas 78712 